\title{
Erosion Profile by a Global Model for Granular Flow
}

\author{
Wen Shen, Tianyou Zhang
}

\begin{abstract}
In this paper we study an integro-differential equation that models the erosion of a mountain profile caused by small avalanches. The equation is in conservative form, with a non-local flux involving an integral of the mountain slope. Under suitable assumptions on the erosion rate, the mountain profile develops several types of singularities, which we call kinks, shocks and hyper-kinks. We study formation of these singularities, and derive admissibility conditions. Furthermore, entropy weak solutions to the Cauchy problem are constructed globally in time, taking limits of piecewise affine approximate solutions. Entropy and entropy flux functions are introduced, and Lax entropy condition is established for the weak solutions.
\end{abstract}

\section{Introduction}

On the real line, consider the integro-differential equation

$$
u_{t}-\left(\exp \int_{x}^{\infty} f\left(u_{x}(t, y)\right) d y\right)_{x}=0, \quad t \geq 0
$$

together with an initial data

$$
u(0, x)=\bar{u}(x) .
$$

Here the flux is a non-local function. For convenience, we define the flux function

$$
F\left(x ; u_{x}\right) \doteq \exp \int_{x}^{\infty} f\left(u_{x}(t, y)\right) d y .
$$

Although $F$ depends on $u_{x}$ in a non-local way, in the rest of the paper we shall often write $F(x)$, without causing confusion. 
The equation (1.1) describes the erosion of a mountain profile by small avalanches. Here $t$ is time, $x$ is the one-dimensional space variable, and $u=u(t, x)$ describes the height of the mountain. We always assume that the slope has positive sign: $u_{x}(0, x)>0$. We also assume that there is a (normalized) equilibrium slope $u_{x}=1$ such that $f(1)=0$, while $f\left(u_{x}\right)>0$ for $u_{x}>1$ and $f\left(u_{x}\right)<0$ for $u_{x}<1$.

In the above model, granular matter is poured at unit rate from $x=$ $\infty$ (or from an uphill location outside the interval of interest), and slides downhill along the mountain slope, from right to left. As it moves, in regions where the mountain slope $u_{x}$ is less than 1 , part of this granular matter is deposited, and the size of the avalanche shrinks. On the other hand, in regions where the slope is larger than 1 , the size of the avalanche grows, putting more granular matter in motion (see Figure 1).

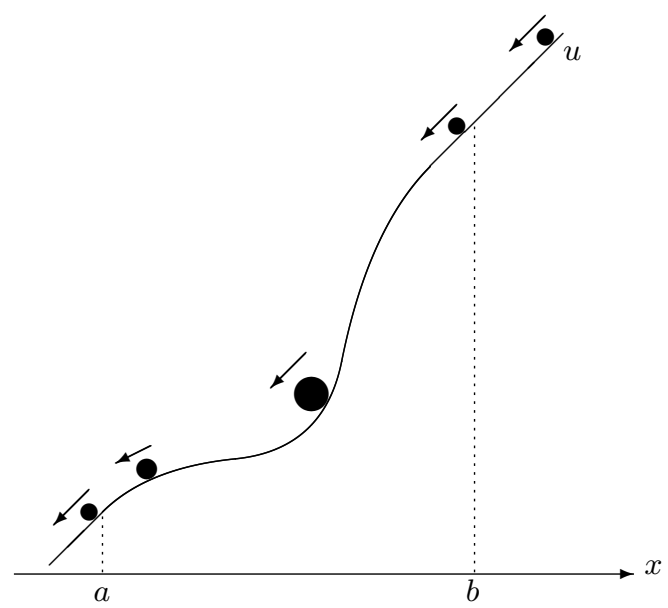

Fig. 1. The size of an avalanche grows when the slope of the mountain is $u_{x}>1$, and shrinks when $u_{x}<1$.

The model (1.1) can be formally derived as follows. Let $u=u(t, x)$ be the height of a mountain profile, on top of which there is an additional layer of sliding material, of thickness $h(t, x)$. We assume that there exist functions (depending only on the mountain slope)

$$
\begin{aligned}
& \alpha\left(u_{x}\right): \text { speed of the moving layer, } \alpha\left(u_{x}\right)>0, \\
& \beta\left(u_{x}\right) \text { : erosion rate per unit moving mass per unit time. }
\end{aligned}
$$

In other words, $\alpha\left(u_{x}\right)$ is the speed at which matter moves down the slope, toward the left. Moreover, a unit amount of sliding mass puts into motion an additional amount $\beta\left(u_{x}\right)$ of mass, per unit time. As a result, a unit amount of moving sand puts into motion an additional amount

$$
f\left(u_{x}\right) \doteq \frac{\beta\left(u_{x}\right)}{\alpha\left(u_{x}\right)}
$$


of sand, per unit length of distance covered.

A mass $m_{b}$ of sand pouring across a point $b$ grows larger or smaller as it traverses the interval $[a, b]$, depending on the slope $u_{x}$ on this interval. As it reaches $a$, its size is

$$
m_{a}=\left(\exp \int_{a}^{b} f\left(u_{x}(y)\right) d y\right) m_{b} .
$$

Hence

$$
\begin{aligned}
& \frac{d}{d t}\left(\int_{a}^{b} u(t, x) d x\right)=m_{b}-m_{a} \\
= & \exp \int_{b}^{\infty} f\left(u_{x}(y)\right) d y-\exp \int_{a}^{\infty} f\left(u_{x}(y)\right) d y=F\left(b ; u_{x}\right)-F\left(a ; u_{x}\right) .
\end{aligned}
$$

This is the integral form of the conservation equation for $u$, with flux $F$. Since $a, b$ are arbitrary, this formally leads to the integro-differential equation (1.1).

In the case where

$$
\alpha\left(u_{x}\right)=u_{x}, \quad \beta\left(u_{x}\right)=u_{x}-1, \quad f\left(u_{x}\right)=\frac{u_{x}-1}{u_{x}},
$$

the model (1.1) was derived in [2] as the slow erosion limit for a system of $2 \times 2$ balance laws describing granular flow in one space dimension:

$$
\left\{\begin{array}{l}
h_{t}=\left(h u_{x}\right)_{x}-\left(1-u_{x}\right) h, \\
u_{t}=\left(1-u_{x}\right) h .
\end{array}\right.
$$

Here $u$ and $h$ are the heights of the standing and moving layers, respectively. It was observed that the evolution of the mountain profile in the slow erosion limit actually depends only on the total mass being poured from the top, not on the pouring rate. In this paper we shall assume that this rate is $\equiv 1$, so that the time $t$ actually equals to the total amount of mass being poured from the top, and we will still use $t$ as independent variable.

The model (1.7) was first proposed in [18]. It is a nonlinear $2 \times 2$ system of conservation laws, providing an approximate description of the evolution of two layers of granular matter (a standing and a moving layer). A mathematical analysis of steady state solutions for (1.7) was carried out in [11, 12]; a numerical study has been performed in [17].

More recently, the first author and collaborators studied analytical properties of time dependent solutions of (1.7). In [24] an existence result for global smooth solutions is proved. The paper [1] establishes the global existence to the Cauchy problem with large data, within a class of functions with bounded variation. Furthermore, in [2] the authors prove the global existence of large BV solutions for an initial-boundary value problem. They also show that, as the thickness of the moving layer becomes very thin, 
the limit profile of the standing layer is described by the scalar integrodifferential equation in (1.1) with $f$ as in (1.6). For the class of functions $f$ with sub-linear growth, the slope $p=u_{x}$ does not blow up, and differentiating (1.1) gives a integro-differential conservation law for $p$. In this case, the well-posedness of the Cauchy problem is studied in [4], and an initial-boundary value problem in [3].

In the present paper we study (1.1) for a wider class of functions $f$, leading to the formation of different types of singularities. On the function $f$ we make the following assumptions.

(A) The function $f:] 0,+\infty\left[\mapsto \mathbb{R}\right.$ is in $\mathcal{C}^{2}$, with

$$
f^{\prime}(w) \geq \eta_{0}>0, \quad f^{\prime \prime}(w) \leq 0, \quad f(1)=0, \quad \lim _{w \rightarrow 0+} f(w)=-\infty .
$$

Furthermore, $f$ has a linear asymptote

$$
\lim _{w \rightarrow+\infty} f(w)-w f^{\prime}(w)<\infty .
$$

The assumptions (A) imply that $f^{\prime}$ has a well-defined limit at $w \rightarrow+\infty$,

$$
\eta_{0} \doteq \lim _{w \rightarrow+\infty} f^{\prime}(w)>0 .
$$

This implies that $f(w)$ grows at a linear rate for large $w$. Therefore, the following integral is bounded

$$
\int_{2}^{\infty} \frac{1}{f^{2}(w)} d w<\infty
$$

Under the above assumptions, we prove that the mountain profile $u(\cdot)$ can exhibit three types of singularities:

- Kinks, where the mountain profile is continuous but its slope has distinct right and left limits: $0<u_{x}(x-)<u_{x}(x+)<\infty$.

- Hyperkinks, where the mountain profile $u$ is continuous but its slope has an infinite limit from the right (and a finite or infinite limit from the left): $u_{x}(x+)=+\infty$.

- Jumps, where the mountain profile has an upward discontinuity, i.e., $u(x-)<u(x+)$.

We seek BV solutions for the Cauchy problem, on a bounded interval of interests. It would be also desirable to have some form of control over the total variation for $u_{x}$ since it is the independent variable for the function $f$. But this is not possible because we consider solutions with infinite slope. Fortunately, it is possible to control the total variation of the characteristic speed $f^{\prime}\left(u_{x}\right) F$, or $f^{\prime}\left(u_{x}\right)$. In fact, observe that $f^{\prime}\left(u_{x}\right)$ changes very little for 
large $u_{x}$ and approaches $\eta_{0}$ as $u_{x} \rightarrow+\infty$. This motivates the definition of the following auxiliary function

$$
\zeta(w) \doteq \begin{cases}\frac{w-2}{f^{2}(2)}, & \text { if } w<2, \\ \int_{2}^{w} \frac{1}{f^{2}(s)} d s, & \text { if } w \geq 2 .\end{cases}
$$

We see that $\zeta(w)$ is a strictly increasing function, and uniformly bounded thanks to (1.11). Furthermore, $\zeta^{\prime}(w)$ is continuous and decreasing, and $\zeta^{\prime}(w) \rightarrow 0$ as $w \rightarrow+\infty$. These are very similar properties as for $f^{\prime}$. As we will see later, $\zeta$ is more flexible and easier to control. This function will also be used in the definition of the entropy functions.

We note that $u(x)=x+c$ with any constant $c$ is an equilibrium solution. We define the deviation function $U(x)$ that describes the deviation from equilibrium for $u$

$$
U(x) \doteq u(x)-x .
$$

Solutions of the Cauchy problem will be obtained within the class $\mathcal{W}$ consisting of all functions $u: \mathbb{R} \mapsto \mathbb{R}$ satisfying the following properties:

$\left(W_{1}\right)$ There exists a constant $\kappa_{0}>0$ such that

$$
u(x)-u(y) \geq \kappa_{0}(x-y), \quad \forall x>y .
$$

$\left(W_{2}\right)$ There exists an interval $I=[a, b]$ such that

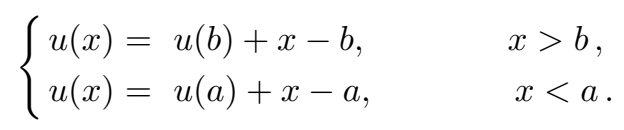

$\left(W_{3}\right)$ The total variation of the deviation function $U(x) \doteq u(x)-x$ is bounded, i.e.,

$$
\operatorname{TV}\{U(\cdot)\}<\infty
$$

$\left(W_{4}\right)$ By setting

$$
\zeta\left(u_{x}(x)\right)=\zeta(+\infty) \doteq \lim _{w \rightarrow+\infty} \int_{2}^{w} \frac{1}{f^{2}(s)} d s
$$

for $x$ in the support of the singular part of the measure $D_{x} u$, the function $x \mapsto \zeta\left(u_{x}(x)\right)$ has bounded variation.

A definition of entropy weak solutions for (1.1)-(1.2) is now given.

Definition 1. A function $u:[0, T] \times \mathbb{R} \mapsto \mathbb{R}$ is called an entropy weak solution for (1.1) with initial data $\bar{u}(x) \in \mathcal{W}$ if $u(0, x)=\bar{u}(x)$, and

$-u(t, \cdot) \in \mathcal{W}$ for every $t \in[0, T]$. 
- For every test function $\varphi \in \mathcal{C}_{c}^{\infty}\left(\mathbb{R}^{2}\right)$, one has the integral identity

$$
\int_{0}^{T} \int_{\mathbb{R}}\left(u \varphi_{t}-F \varphi_{x}\right) d x d t=\int_{\mathbb{R}}(u(T, x) \varphi(T, x)-u(0, x) \varphi(0, x)) d x .
$$

- (Entropy conditions). For almost all $0<t \leq T$, the Lax entropy condition is satisfied at every singularity (kink, hyper-kink and shock). Let $\lambda(t)$ denote the speed of the singularity, and let

$$
\lambda^{-}(t) \doteq f^{\prime}\left(u_{x}(t, x-)\right) F\left(x-; u_{x}\right), \quad \lambda^{+} \doteq f^{\prime}\left(u_{x}(t, x+)\right) F\left(x+; u_{x}\right)
$$

be the characteristic speed to the left and right side of the singularity, respectively. Then

$$
\lambda^{-}(t) \geq \lambda(t) \geq \lambda^{+}(t)
$$

Piecewise affine approximations (with discontinuities) for $u(t, \cdot)$ will be constructed by an algorithm that traces the fronts, both their positions and their point values. The algorithm determines a set of ODEs for the evolution of the nodal points $x_{i}(t)$ and the nodal values $u_{i}(t)=u\left(x_{i}(t)\right)$.

Note that $x \mapsto u_{x}(t, x)$ is a measure-valued function, and the flux $F$ contains an integral of $f\left(u_{x}\right)$. The convergence of the flux is not straightforward. By condition $\left(W_{1}\right)$, the map $x \mapsto u(x)$ is strictly increasing. Because of the lower bound on $u_{x}$, the inverse map $u \mapsto x(u)$ is uniformly Lipschitz. It is convenient to make a variable change and use the height $u$ as independent variable instead of $x$ in the definition of $F$. Therefore, we define

$$
F\left(u ; u_{x}\right) \doteq \exp \left\{\int_{u}^{\infty} g\left(u_{x}(t, v)\right) d v\right\}
$$

where

$$
g(w) \doteq \begin{cases}\frac{f(w)}{w}, & \text { if } w<\infty, \\ \lim _{w \rightarrow \infty} \frac{f(w)}{w}=\lim _{w \rightarrow \infty} f^{\prime}(w)=\eta_{0}, & \text { if } \quad w=\infty .\end{cases}
$$

The function $w \mapsto g(w)$ is uniformly Lipschitz on the interval $\left[\kappa_{0}, \infty\right]$ with $\kappa_{0}>0$. Therefore the map $u \mapsto F\left(u ; u_{x}\right)$ is uniformly Lipschitz continuous. An a priori BV estimate on the flux $F$ can then be used to establish the compactness of a sequence of approximate solutions. Our main theorem is stated below.

Theorem 1. Let $T>0$ and an initial data $\bar{u} \in \mathcal{W}$ be given. Then the Cauchy problem (1.1)-(1.2) admits an entropy weak solution $u=u(t, x)$ defined for all $t \in[0, T]$.

Other PDE models for granular flows can be found in $[5,23]$ and in the book [16]. An extension of the model (1.7) was proposed in the recent paper [13], introducing an additional equation for the velocity of the sliding 
material. This leads to a refined but more complicated description $(\mathrm{a} 3 \times 3$ system) of the complete dynamics.

Several other examples of scalar conservation laws with integro differential terms have been considered in the literature, and are worth mentioning here. In particular, the variational wave equation

$$
u_{t}+f(u)_{x}=\int_{0}^{x} f^{\prime \prime}(u) u_{x}^{2} d y, \quad x>0
$$

was studied in $[9,19]$, while the Camassa-Holm equation

$$
u_{t}+\left(\frac{u^{2}}{2}\right)_{x}+\left(\frac{1}{2} \int_{-\infty}^{\infty} e^{-|x-y|}\left(u^{2}(y)+\frac{u_{x}^{2}(y)}{2}\right) d y\right)_{x}=0
$$

was analyzed in a large number of papers (e.g. $[10,7,8]$ and the reference therein). In both cases, writing a balance law for the quantity $u_{x}^{2}$ one obtains the a priori bound

$$
\left\|u_{x}(t, \cdot)\right\|_{\mathbf{L}^{2}} \leq C .
$$

In turn, this implies that the solution $u(t, \cdot)$ remains Hölder continuous at all times.

In contrast, solutions $u(t, x)$ to our equation (1.1) may well develop jumps, so that the distributional derivative $u_{x}$ contains point masses. However, if the function $f$ has linear growth and satisfies the assumptions (A), we show that the Cauchy problem has well defined solutions globally in time.

For the approximate solutions and their a priori estimates, we further require a decay property for the second derivative of $f$. Namely, we assume

$$
\sup _{w \geq \kappa_{0}}\left|f^{\prime \prime}(w)\right| w^{3}<\infty .
$$

This requirement implies an asymptote for $f$, therefore is stronger than (1.9). This restriction will be dropped later.

The remainder of the paper is structured in the following way. Section 2 contains some basic analysis. Formation of singularities is studied by the method of characteristics. Admissibility conditions, wave speeds and wave interactions are also studied. In Section 3 we provide some technical lemmas, derive formal bounds and introduce entropy and entropy flux pairs. In Section 4 we construct a piecewise affine approximate solution to the Cauchy problem. Assuming in addition (1.21), in Section 5, suitable a priori estimates for the approximate solution are established. As customary, the limit of a convergent subsequence then provides an entropy weak solution, which is completed in Section 6. We then drop the assumption (1.21) at the end of Section 6 . We end the paper with some concluding remarks. 


\section{Basic Analysis}

We first note that we have

$$
F_{x}=-f F=-f\left(u_{x}(t, x)\right) \cdot \exp \int_{x}^{\infty} f\left(u_{x}(t, y)\right) d y .
$$

For smooth solutions, setting $w \doteq u_{x}$ we find

$$
w_{t}-\left(F_{x}\right)_{x}=w_{t}+(f F)_{x}=0 .
$$

As long as the solution remains smooth, the method of characteristics yields

$$
\left\{\begin{array}{l}
\dot{x}=F(x ; w) f^{\prime}(w), \\
\dot{u}=\left(f^{\prime}(w) w-f(w)\right) F(x ; w), \\
\dot{w}=F(x ; w) f^{2}(w),
\end{array}\right.
$$

with initial data

$$
x(0)=x_{0}, \quad u\left(0, x_{0}\right)=\bar{u}\left(x_{0}\right), \quad w\left(0, x_{0}\right)=\bar{u}_{x}\left(x_{0}\right) .
$$

Of course, (2.3) is not a closed equation, because the flux $F$ depends on the entire profile of the function $w$ on the half line $[x,+\infty[$.

\subsection{Formation of singularities: kinks and shocks.}

The slope $w$ satisfies the conservation law (2.2), where the local flux $f$ is non-linear. By this non-linearity, the characteristics will cross, forming discontinuities in $w$. These will result in kinks in the mountain profile, therefore are weak singularities, and we call them kinks. To study the development of kinks, we set $z \doteq w_{x}=u_{x x}$. Differentiating (2.2) in $x$ we find the evolution of $z$ along a characteristic curve

$$
\frac{d}{d t} z(t, x(t))=z_{t}+F f^{\prime}(w) z_{x}=-F f^{\prime \prime}(w) z^{2}+3 F f f^{\prime} z-F f^{3}(w) .
$$

As long as $w$ remains finite, when $z$ is large, the leading term is $-F f^{\prime \prime}(w) z^{2}$. Hence, by the assumptions $f^{\prime \prime}<0$ and $F>0$, the function $z(t, x(t))$ can blow up to $+\infty$ in finite time:

$$
z(t, x(t)) \rightarrow+\infty, \quad t \rightarrow T-.
$$

In other words, a convex kink will develop in the solution $u$. However, concave kinks will not form, because for $z$ with large negative value, the first term $-F f^{\prime \prime}(w) z^{2}$ will dominate with positive value, and $z$ will increase.

The strong singularities in the solution are discontinuities in the profile variable $u$, and we call these shocks. From the 3rd equation in (2.3), the slope is non-decreasing along characteristics. We assume that the flux remains uniformly positive $F \geq F^{0}>0$. As long as the solution remains smooth, (i.e., no kinks yet), the following cases can occur: 
(a) If $0<w(0) \leq 1$, then $w(t) \rightarrow 1$ as $t \rightarrow \infty$.

(b) If $w(0)>1$, since $\int_{2}^{+\infty} f^{-2}(w) d w<\infty$, then the slope $w$ will blow up in finite time. Shocks could be formed in the solutions of $u$.

Remark 1. If $w$ blows up to infinite value, them the last (negative) term $-F f^{3}(w)$ in (2.5) could dominate, resulting in a concave kink. This would be attached to the right side of a shock.

Remark 2. Observe that

$$
\dot{u}=-\frac{d}{d w}\left(\frac{w}{f(w)}\right) \cdot f^{2}(w) F=-\frac{d}{d w}\left(\frac{w}{f(w)}\right) \cdot \dot{w},
$$

which implies that the quantity $u=w / f(w)$ remains constant along characteristics. During blowup, the change in $u$ should remain finite since erosion should not produce an infinite deep hole. Therefore, the following must hold during blowup

$$
\lim _{w \rightarrow+\infty} \frac{w}{f(w)}<\infty .
$$

Then, $f(w)$ must grow at least at a linear rate for large $w$. In fact, this is observed in [3,4]. When (2.6) fails, i.e., if one has instead

$$
\lim _{w \rightarrow+\infty} \frac{f(w)}{w}=\lim _{w \rightarrow+\infty} f^{\prime}(w)=0,
$$

it is shown in $[3,4]$ that $u_{x}$ remains uniformly bounded for all time. The only singularities in the solution are the kinks in the mountain profile.

Therefore, the assumption

$$
\lim _{w \rightarrow+\infty} f^{\prime}(w)=\eta_{0}>0
$$

is the precise condition for possible blowup of slope.

\subsection{Wave speeds and their admissibility conditions.}

A kink in $u$ corresponds to a jump in the slope $w$. The slope $w$ satisfies the equation (2.2). Consider a jump in $w$ at $x_{0}$, with $w^{-}, w^{+}$as the left and right state (resp.). By the definition, the flux $F$ will be continuous at $x_{0}$. By the Rankine-Hugoniot condition one finds the kink speed

$$
\lambda_{k}=\frac{f\left(w^{+}\right)-f\left(w^{-}\right)}{w^{+}-w^{-}} F\left(x_{0}\right) .
$$

Since $f$ is concave, only upward jumps are admissible, i.e., $w^{+}>w^{-}$.

The shock speed is derived from the Rankine-Hugoniot equation in the $u$ variable. Assume that $u$ has a jump at $x=x_{0}$. Consider the right and left limits

$$
u^{+} \doteq \lim _{x \rightarrow x_{0}+} u(x), \quad u^{-} \doteq \lim _{x \rightarrow x_{0}-} u(x)
$$


We shall also need the right and left limits of the flux function $F(x)$, as $x \rightarrow x_{0}$. To compute this, we can approximate the function $u$ with functions $u^{(\epsilon)}$ such that

- $u^{(\epsilon)}(x)=u(x)$ for $\left|x-x_{0}\right| \geq \epsilon$,

- $u^{(\epsilon)}$ has piecewise constant slope on the interval $\left[x_{0}-\epsilon, x_{0}+\epsilon\right]$.

By the assumptions (A), we then have

$$
\begin{aligned}
\lim _{\epsilon \rightarrow 0} \int_{x_{0}-\epsilon}^{x_{0}+\epsilon} f\left(u_{x}^{(\epsilon)}(y)\right) d y & =\lim _{\epsilon \rightarrow 0} \int_{x_{0}-\epsilon}^{x_{0}+\epsilon} f\left(\frac{u\left(x_{0}+\epsilon\right)-u\left(x_{0}-\epsilon\right)}{2 \epsilon}\right) d y \\
& =\eta_{0}\left(u^{+}-u^{-}\right) .
\end{aligned}
$$

Since the size of the shock appears as a parameter, let's define

$$
[u] \doteq u^{+}-u^{-} \geq 0 .
$$

Then

$$
F\left(x_{0}-\right)=F\left(x_{0}+\right) \cdot \exp \left\{\eta_{0}[u]\right\} .
$$

By the Rankine-Hugoniot equation we get the shock speed

$$
\begin{aligned}
\lambda_{s} & =-\frac{F\left(x_{0}+\right)-F\left(x_{0}-\right)}{u^{+}-u^{-}}=\frac{\exp \left\{\eta_{0}[u]\right\}-1}{[u]} F\left(x_{0}+\right) \\
& =\frac{1-\exp \left\{-\eta_{0}[u]\right\}}{[u]} F\left(x_{0}-\right) .
\end{aligned}
$$

The standard Lax [20] admissibility condition applies to shocks. We write $w^{-}, w^{+}$as the left and right state (resp.) for $w$ at $x_{0}$. Let

$$
\begin{aligned}
& \lambda^{-}=F\left(x_{0}-\right) f^{\prime}\left(w^{-}\right)=F\left(x_{0}+\right) \cdot \exp \left\{\eta_{0}[u]\right\} f^{\prime}\left(w^{-}\right), \\
& \lambda^{+}=F\left(x_{0}+\right) f^{\prime}\left(w^{+}\right)
\end{aligned}
$$

be the characteristic speeds to the left and right of the shock, respectively. Note that this is different from a standard conservation law, where the characteristic speeds would depend on $u\left(x_{0} \pm\right)$, not $u_{x}\left(x_{0} \pm\right)$. Lax condition states

$$
\lambda^{-} \geq \lambda_{s} \geq \lambda^{+}
$$

We first claim that $\lambda^{-} \geq \lambda_{s}$ always holds. Indeed, we need to show that

$$
\exp \left\{\eta_{0}[u]\right\} f^{\prime}\left(w^{-}\right) \geq \frac{\exp \left\{\eta_{0}([u])\right\}-1}{[u]}
$$

Note that $[u] \geq 0$ and $\eta_{0} \geq 0$, so (2.13) follows from the fact that $f^{\prime}\left(w^{-}\right) \geq$ $\eta_{0}$ and that $x e^{x} \geq e^{x}-1$ for any $x \geq 0$.

For the condition $\lambda_{s} \geq \lambda^{+}$, we need

$$
\frac{\exp \left\{\eta_{0}[u]\right\}-1}{[u]} \geq f^{\prime}\left(w^{+}\right) .
$$


This condition can be viewed in two ways. Given the size of the shock $[u]$, (2.14) gives a condition on the slope $w^{+}$, namely

$$
w^{+} \geq \gamma, \quad \text { where } \gamma \text { satisfies } f^{\prime}(\gamma)=\frac{\exp \left\{\eta_{0}[u]\right\}-1}{[u]} .
$$

On the other hand, given the slope on the right $w^{+},(2.14)$ provides a condition on the size of the jump. In fact, expand the left of (2.14),

$$
\frac{\exp \left\{\eta_{0}[u]\right\}-1}{[u]}=\eta_{0}+\frac{1}{2} \eta_{0}^{2}[u]+\cdots
$$

and one gets

$$
[u] \geq \frac{2}{\eta_{0}^{2}} \cdot\left(f^{\prime}\left(w^{+}\right)-\eta_{0}\right) .
$$

In summary, for a shock to be admissible, it has to be large enough for fixed slope on the right, or the slope on the right should be large enough for a fixed shock size.

Remark 3. An intuitive physical meaning for the admissibility condition (2.16) can be the following. If the jump is big, then as the avalanche passes through the jump, it flows through a longer path straight down, and causes more erosion, so the slope could remain vertical. On the other hand, if the jump is small, there would be less erosion at the jump, not enough to keep up the vertical slope. As a result, the corner will be smoothed out, resulting in a rarefaction fan.

\subsection{Bridging the two singularities: hyper-kinks.}

We observe that by the admissibility condition (2.15), smaller shocks require steeper slope on the right of the shock. In the limit as $[u] \rightarrow 0$, in (2.15) we have $f^{\prime}(\gamma) \rightarrow \eta_{0}$, therefore we must have $u_{x}^{+} \rightarrow+\infty$, i.e., the slope on the right needs to blow up. This corresponds to a kink with an infinitely large slope on the right. This is a special type of singularity, and we refer to it as hyper-kink. The wave speed, obtained as the limit of the kink speed in $(2.7)$, is

$$
\lim _{w^{+} \rightarrow \infty} \lambda_{k}=\lim _{w^{+} \rightarrow \infty} \frac{f\left(w^{+}\right)-f\left(w^{-}\right)}{w^{+}-w^{-}} F\left(x_{0}\right)=\eta_{0} F\left(x_{0}\right),
$$

and obtained as the limit of the shock speed (2.11), is

$$
\lim _{[u] \rightarrow 0} \lambda_{s}=\lim _{[u] \rightarrow 0} \frac{\exp \left\{\eta_{0}[u]\right\}-1}{[u]} \cdot F\left(x_{0}\right)=\eta_{0} F\left(x_{0}\right) .
$$

They are the same. Therefore, the speed of a hyper-kink at $x_{0}$ is

$$
\lambda_{h k}=\eta_{0} F\left(x_{0}\right) .
$$

In summary, we have 3 types of singularities: kinks, shocks and hyperkinks. See Figure 2 for an illustration. 


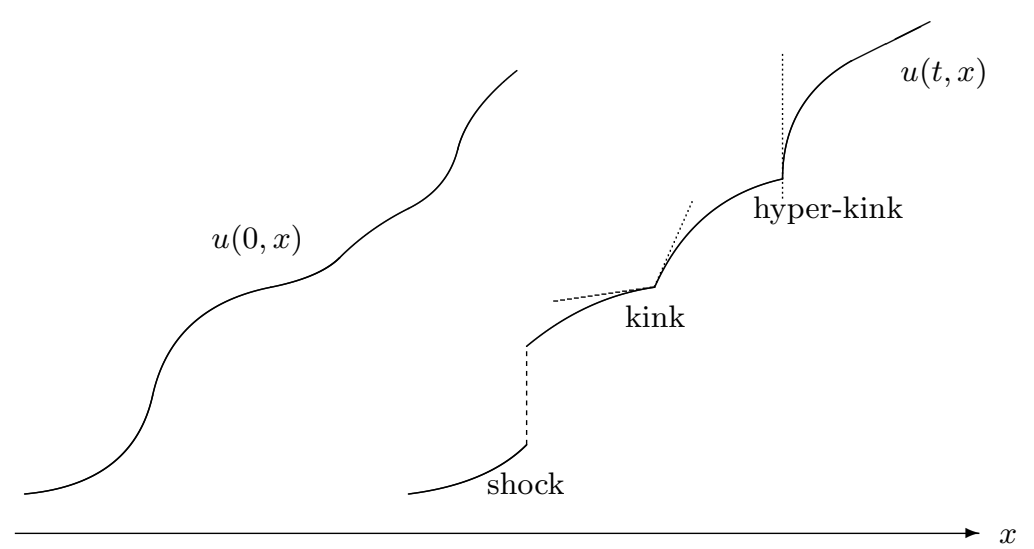

Fig. 2. Three types of singularities: kinks, shocks and hyper-kinks.

\subsection{The generalized Riemann problem and wave interactions.}

The generalized Riemann problem, with a jump in $u$ at some point $x_{0}$, depends only on 4 parameters: $[u], u_{x}^{-}, u_{x}^{+}$and $F^{+}$. The last parameter $F^{+}$ only gives a change of the wave speed, not the qualitative behavior. If the jump is admissible, it will travel as a shock. If the jump is not admissible, i.e., the slope to the right is not large enough to satisfy (2.15), then the solution will have a rarefaction wave merging to the right, with limit slope at the right satisfying the admissible condition (2.15). See Figure 3 for an illustration.

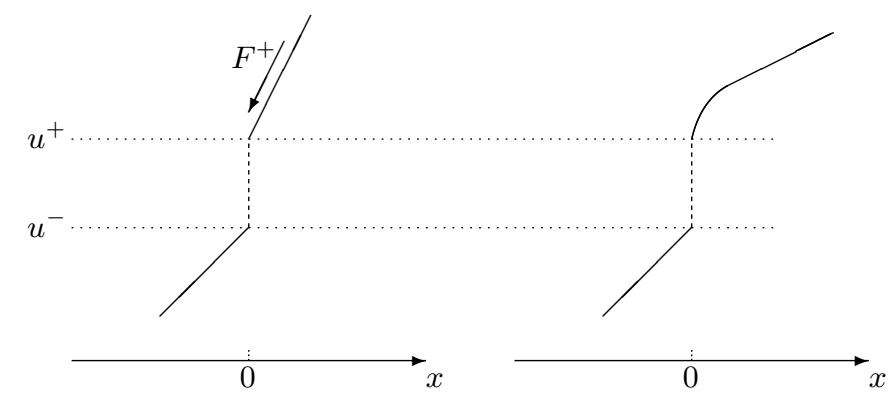

Fig. 3. A generalized Riemann problem for (1.1). Here the data are the left and right limits $u^{-}, u^{+}$of the mountain profile, the limits $u_{x}^{-}, u_{x}^{+}$of the slope, and the incoming flux $F^{+}$.

Note that this is not the regular Riemann problem, and the solution is not self-similar. This analysis gives us an instantaneous behavior of the solution.

There are several types of singularity interactions, which we discuss below.

(a). A kink (or hyper-kink) interacts with a kink (or a hyper-kink). In this case, they will merge into a larger kink (or hyper-kink if one of the incoming 
wave is a hyper-kink). If the new wave is a hyper-kink, it will travel with the hyper-kink speed, and if it is a kink, it travels with the kink speed. For the later case, let $w^{l}, w^{m}, w^{r}$ be the left, middle and right slope before they merge. The speeds of the two kinks before merging are

$$
\lambda_{k}^{l}=F \frac{f\left(w^{m}\right)-f\left(w^{l}\right)}{w^{m}-w^{l}}, \quad \lambda_{k}^{r}=F \frac{f\left(w^{r}\right)-f\left(w^{m}\right)}{w^{r}-w^{m}},
$$

and the speed of the new kink will be

$$
\lambda_{k}^{n}=F \frac{f\left(w^{r}\right)-f\left(w^{l}\right)}{w^{r}-w^{l}} .
$$

Clearly we have $\lambda_{k}^{l}>\lambda_{k}^{n}>\lambda_{k}^{r}$, i.e., the new kink speed lies between the speeds of the two merging kinks.

(b). A shock interacts with a shock. This will result in a bigger shock. Since the larger jumps are "more admissible", the new shock will be admissible. Concerning its speed, let $[u]^{l},[u]^{r}$ be the sizes of the two jumps, and let $F^{m}, F^{r}$ be the flux at middle and right of the two shocks. Then, the speed of the two merging shocks are

$$
\lambda_{s}^{l}=F^{m} \frac{\exp \left\{\eta_{0}[u]^{l}\right\}-1}{[u]^{l}}=F^{r} \exp \left\{\eta_{0}[u]^{r}\right\} \frac{\exp \left\{\eta_{0}[u]^{l}\right\}-1}{[u]^{l}},
$$

and

The speed of the new shock is

$$
\lambda_{s}^{r}=F^{r} \frac{\exp \left\{\eta_{0}[u]^{r}\right\}-1}{[u]^{r}} .
$$

$$
\lambda_{s}^{n}=F^{r} \frac{\exp \left\{\eta_{0}\left([u]^{l}+[u]^{r}\right)\right\}-1}{[u]^{l}+[u]^{r}}=\frac{\lambda_{s}^{l} \cdot[u]^{l}+\lambda_{s}^{r} \cdot[u]^{r}}{[u]^{l}+[u]^{r}},
$$

which is a convex combination of the speeds of the two merging shocks.

(c). A kink (or hyper-kink) interacts with a shock. If the kink is on the left, since the left side of the shock is always admissible, the kink will simply merge into the shock. If the kink is on the right, since the kink is always convex, this would only increase the slope on the right of the shock, keeping it admissible. As a result, the kink would merge into the shock.

In conclusion, when two singularities of the same type interact, they merge into one; When two singularities of different types interact, the weaker singularity merges into the stronger one. If more than two singularities interact, by induction they will merge into one singularity, taking the same type as the strongest singularity among the incoming waves. Therefore, all interactions behave in a similar way as shock interactions for scalar conservation law, and no new singularities are generated at interactions.

By the Lax condition we impose, characteristics will merge into convex kinks from both side. They will also merge into shocks from the left. However, on the right of a shock, characteristic curves could depart from the shock, tangent to the shock curve, at a time $t>0$. It is unclear if an Oleinik type decay estimate [22] would hold. 


\section{Some technical lemmas and formal bounds}

Towards the study of the initial value problem (1.1)-(1.2), we begin with some technical Lemmas and some formal arguments for smooth solutions and entropy inequality. Throughout the paper, we will use $C$ repeatedly, with or without indices, to denote some positive bounded constant that does not depend on the critical variables. The dependence of the constant will be explain immediately after its introduction if it is not obvious.

\subsection{Technical Lemmas}

We first show that the assumption (1.21) implies an asymptote for $f$.

Lemma 1. Let $f$ satisfies the assumptions (1.8) and (1.21). Then, $f^{\prime}$ has the following decay property

$$
\sup _{w \geq \kappa_{0}>0} w^{2}\left(f^{\prime}(w)-\eta_{0}\right)<\infty, \quad \text { where } \eta_{0}=\lim _{w \rightarrow+\infty} f^{\prime}(w) .
$$

This implies that $f$ approaches an asymptote

$$
\lim _{w \rightarrow+\infty}\left|f(w)-w \eta_{0}\right|<\infty
$$

Proof. The property (3.1) follows because

$$
\begin{aligned}
\left|f^{\prime}(w)-\eta_{0}\right| & \leq \int_{w}^{\infty}\left|f^{\prime \prime}(s)\right| d s=\int_{w}^{\infty} s^{3}\left|f^{\prime \prime}(s)\right| \frac{1}{s^{3}} d s \\
& \leq \frac{1}{w^{2}} \sup _{s \geq \kappa_{0}}\left|s^{3} f^{\prime \prime}(s)\right|
\end{aligned}
$$

This indicates that $\left(f^{\prime}(w)-\eta_{0}\right)$ is absolutely integrable. Furthermore, for any $w, \bar{w} \geq \kappa_{0}$, we have

$$
\begin{aligned}
\left(f(w)-w \eta_{0}\right)-\left(f(\bar{w})-\bar{w} \eta_{0}\right) & =\int_{\bar{w}}^{w} \frac{d}{d s}\left(f(s)-s \eta_{0}\right) d s \\
& =\int_{\bar{w}}^{w}\left(f^{\prime}(s)-\eta_{0}\right) d s<\infty .
\end{aligned}
$$

Letting $w \rightarrow \infty$, we get (3.2). 
Remark 4. As immediate consequences of Lemma 1 and the property (A), the following bounds also hold.

$$
\begin{array}{r}
\lim _{w \rightarrow+\infty}\left|f(w)-w f^{\prime}(w)\right|<\infty . \\
\sup _{w \geq \kappa_{0}} f^{3}(w)\left|f^{\prime \prime}(w)\right|<\infty, \\
\sup _{w \geq \kappa_{0}} f^{2}(w)\left(f^{\prime}(w)-\eta_{0}\right)<\infty .
\end{array}
$$

Thanks to the decay property of $f^{\prime \prime}$ in (1.21), the changes in $f^{\prime}$ and $g$ are controlled by the change in $\zeta$. This is the content of next Lemma.

Lemma 2. Let $f$ satisfy $(\boldsymbol{A})$ and (1.21). For any $\tilde{w} \geq \bar{w} \geq \kappa_{0}>0$, we have

$$
\begin{aligned}
& f^{\prime}(\bar{w})-f^{\prime}(\tilde{w}) \leq C[\zeta(\tilde{w})-\zeta(\bar{w})], \\
& |g(\tilde{w})-g(\bar{w})| \leq C|\zeta(\tilde{w})-\zeta(\bar{w})|,
\end{aligned}
$$

where $C$ is some constant that depends only on $\kappa_{0}$ and properties of $f$.

Proof. Consider $f^{\prime}(w)$ as a function of $\zeta(w)$. By the Chain Rule we have

$$
\frac{d\left(f^{\prime}\right)}{d \zeta}=\frac{d\left(f^{\prime}\right) / d w}{d \zeta / d w}=\frac{f^{\prime \prime}(w)}{\zeta^{\prime}(w)}= \begin{cases}f^{\prime \prime}(w) f^{2}(2), & w \leq 2 \\ f^{\prime \prime}(w) f^{2}(w), & w>2\end{cases}
$$

which is strictly negative and uniformly bounded for $w \geq \kappa_{0}$ by (3.4). The property (3.6) follows by the Mean Value Theorem.

In a similar way, consider $g(w)$ as a function of $\zeta(w)$, we have

$$
\frac{d g}{d \zeta}=\frac{d g / d w}{d \zeta / d w}=\frac{w f^{\prime}(w)-f(w)}{w^{2} \zeta^{\prime}(w)}=\left\{\begin{array}{l}
\frac{w f^{\prime}(w)-f(w)}{w^{2}} f^{2}(2), \quad w \leq 2, \\
\left(w f^{\prime}(w)-f(w)\right) \frac{f^{2}(w)}{w^{2}}, w>2 .
\end{array}\right.
$$

Thanks to (3.3), this is uniformly bounded for all $w \geq \kappa_{0}$. Again, (3.7) follows from the Mean Value Theorem.

Furthermore, the next lemma reveals some more relations between $f$ and $\zeta$, which would be useful to control the error of the approximate solutions.

Lemma 3. Let $f$ satisfy $(\boldsymbol{A})$ and (1.21), and let $\bar{w} \geq 2$ be bounded. For any $w \geq \bar{w}$, we have

$$
\begin{aligned}
& f(\bar{w})\left(f^{\prime}(\bar{w})-\frac{f(w)-f(\bar{w})}{w-\bar{w}}\right) \leq C(\zeta(w)-\zeta(\bar{w})), \\
& f(w)\left(\frac{f(w)-f(\bar{w})}{w-\bar{w}}-f^{\prime}(w)\right) \leq C(\zeta(w)-\zeta(\bar{w})),
\end{aligned}
$$

where $C$ is some constant depending only on properties of $f$, but not on $w$ or $\bar{w}$. 
Proof. These properties clearly holds if $w$ remains bounded. If $w$ blows up, we have

$$
\lim _{w \rightarrow+\infty} f(\bar{w})\left(f^{\prime}(\bar{w})-\frac{f(w)-f(\bar{w})}{w-\bar{w}}\right) \cdot \frac{1}{\zeta(w)-\zeta(\bar{w})}=\frac{f(\bar{w})\left(f^{\prime}(\bar{w})-\eta_{0}\right)}{\zeta(+\infty)-\zeta(\bar{w})} .
$$

To see that the constant $C$ in (3.8) is uniform in $\bar{w}$, we compute

$$
\begin{aligned}
\lim _{\bar{w} \rightarrow+\infty} \frac{f(\bar{w})\left(f^{\prime}(\bar{w})-\eta_{0}\right)}{\zeta(+\infty)-\zeta(\bar{w})} & =\lim _{\bar{w} \rightarrow+\infty} \frac{f^{\prime}(\bar{w})\left(f^{\prime}(\bar{w})-\eta_{0}\right)+f(\bar{w}) f^{\prime \prime}(\bar{w})}{-\zeta^{\prime}(\bar{w})} \\
& =\lim _{\bar{w} \rightarrow+\infty} f^{\prime}(\bar{w}) f^{2}(\bar{w})\left(f^{\prime}(\bar{w})-\eta_{0}\right)+f^{3}(\bar{w}) f^{\prime \prime}(\bar{w})
\end{aligned}
$$

which is uniformly bounded because of (3.5) and (1.21), proving (3.8).

The proof for (3.9) is completely similar. We have

$$
\begin{aligned}
& \lim _{w \rightarrow+\infty} f(w)\left(\frac{f(w)-f(\bar{w})}{w-\bar{w}}-f^{\prime}(w)\right) \\
= & \lim _{w \rightarrow+\infty} \frac{f(w)}{w-\bar{w}}\left(\lim _{w \rightarrow+\infty}\left\{f(w)-f^{\prime}(w) w\right\}-\lim _{w \rightarrow+\infty}\left\{f(\bar{w})-f^{\prime}(w) \bar{w}\right\}\right) \\
= & \eta_{0}\left(M-\left(f(\bar{w})-\eta_{0} \bar{w}\right)\right),
\end{aligned}
$$

where

$$
M=\lim _{w \rightarrow+\infty}\left\{f(w)-f^{\prime}(w) w\right\} .
$$

To prove that the constant $C$ in (3.9) is uniform in $\bar{w}$, we compute

$$
\lim _{\bar{w} \rightarrow+\infty} \eta_{0} \frac{M-\left(f(\bar{w})-\eta_{0} \bar{w}\right)}{\zeta(+\infty)-\zeta(\bar{w})}=\lim _{\bar{w} \rightarrow+\infty} \eta_{0} f^{2}(\bar{w})\left(f^{\prime}(\bar{w})-\eta_{0}\right)<\infty,
$$

thanks to (3.5). This completes the proof.

In next Lemma we list many bounds satisfied by $f$ and $F$ if $u \in \mathcal{W}$.

Lemma 4. Let $u(x) \in \mathcal{W}$ be a function that satisfies the conditions $\left(W_{1}\right)-$ $\left(W_{3}\right)$. Then

$$
\begin{aligned}
& \int_{-\infty}^{\infty}\left|f\left(u_{x}(x)\right)\right| d x \leq C, \\
& \exp \{-C\} \leq F \leq \exp \{C\} \\
& T V\{F\} \leq C \cdot \exp \{C\}
\end{aligned}
$$

where the constant $C$ is

$$
C=f^{\prime}\left(\kappa_{0}\right) \cdot T V\{U\}
$$

Furthermore, if $u$ also satisfies $\left(W_{4}\right)$, then the maps $x \mapsto f^{\prime}\left(u_{x}\right), x \mapsto g\left(u_{x}\right)$ and $x \mapsto f^{\prime}\left(u_{x}\right) F(x)$ all have bounded variation. 
Proof. If $u(x)$ is continuous, then formally we have

$$
\begin{aligned}
& \int_{-\infty}^{\infty}\left|f\left(u_{x}(x)\right)\right| d x=\int_{-\infty}^{\infty}\left|f\left(u_{x}(x)\right)-f(1)\right| d x \\
& =\int_{-\infty}^{\infty}\left|\int_{1}^{u_{x}(x)} f^{\prime}(s) d s\right| d x \leq f^{\prime}\left(\kappa_{0}\right) \int_{-\infty}^{\infty}\left|u_{x}(x)-1\right| d x \\
& =f^{\prime}\left(\kappa_{0}\right) \int_{-\infty}^{\infty}\left|U_{x}\right| d x=f^{\prime}\left(\kappa_{0}\right) \cdot \operatorname{TV}\{U\}
\end{aligned}
$$

and (3.10-3.12) follow.

However $x \mapsto U$ is only $\mathrm{BV}$ and can be discontinuous. To prove the same result, we will use $u$ as the independent variable instead of $x$. The map $u \mapsto U(t, x(t, u))$ is continuous, and the total variation of $U$ equals to the $\mathbf{L}^{1}$ norm of $d U / d u$. Let $u_{a} \doteq u(a)$ and $u_{b} \doteq u(b)$. Since $U$ is BV, then $u_{b}-u_{a}$ is bounded. We have

$$
\operatorname{TV}\{U\}=\int_{u_{a}}^{u_{b}}\left|\frac{d U}{d u}\right| d u=\int_{u_{a}}^{u_{b}}\left|1-\frac{d x}{d u}\right| d u=\int_{u_{a}}^{u_{b}}\left|1-\frac{1}{u_{x}}\right| d u .
$$

Now since $f=0$ outside $[a, b]$, by considering $u_{x}$ as a function of $u$, we have

$$
\begin{aligned}
& \int_{-\infty}^{+\infty}\left|f\left(u_{x}(x)\right)\right| d x=\int_{a}^{b}\left|f\left(u_{x}(x)\right)\right| d x \\
& =\int_{u_{a}}^{u_{b}} \frac{1}{u_{x}(u)}\left|f\left(u_{x}(u)\right)\right| d u=\int_{u_{a}}^{u_{b}} \frac{1}{u_{x}}\left|f\left(u_{x}\right)-f(1)\right| d u \\
& =\int_{u_{a}}^{u_{b}} \frac{1}{u_{x}}\left|\int_{1}^{u_{x}} f^{\prime}(s) d s\right| d u \leq f^{\prime}\left(\kappa_{0}\right) \int_{u_{a}}^{u_{b}} \frac{1}{u_{x}}\left|u_{x}-1\right| d u \\
& =f^{\prime}\left(\kappa_{0}\right) \int_{u_{a}}^{u_{b}}\left|1-\frac{1}{u_{x}}\right| d u=f^{\prime}\left(\kappa_{0}\right) \operatorname{TV}\{U\} .
\end{aligned}
$$

Then, (3.10-3.11) follow. For the total variation of $F$, observing that $u \mapsto$ $F\left(x(t, u) ; u_{x}\right)$ is a Lipschitz continuous map, therefore

$$
\operatorname{TV}\{F\}=\int_{u_{a}}^{u_{b}}\left|F_{u}\right| d u=\int_{u_{a}}^{u_{b}}\left|\frac{f\left(u_{x}\right)}{u_{x}}\right| F d u \leq \int_{u_{a}}^{u_{b}}\left|\frac{f\left(u_{x}\right)}{u_{x}}\right| d u \cdot\|F\|_{\mathbf{L}^{\infty}} .
$$

By applying (3.11) and (3.13), we get (3.12).

As an immediate consequence of Lemma 2, we see that the total variations of $x \mapsto f^{\prime}\left(u_{x}(x)\right)$ and $x \mapsto g\left(u_{x}(x)\right)$ are bounded by the total variation of $x \mapsto \zeta\left(u_{x}(x)\right)$. Combining with the total variation bound on $F$, the characteristic speed $f^{\prime}\left(u_{x}\right) F$ has bounded variation as well. 


\subsection{Formal bounds}

In this subsection we derive some essential formal bounds on smooth solutions. These bounds would provide insight and guideline for the a priori bounds for approximate solutions.

Lemma 5. Let the initial data $\bar{u} \in \mathcal{W}$ be smooth and assume the solution $u(t, x)$ remains smooth, then $t \mapsto u(t, \cdot) \in \mathcal{W}$.

Proof. We will check if all the conditions $\left(W_{1}\right)-\left(W_{4}\right)$ hold.

$\left(W_{1}\right)$. From the equations (2.3) it follows $\dot{w} \geq 0$. Hence the function $t \mapsto$ $\inf _{x} u_{x}(t, x)$ is non-decreasing along solutions of the Cauchy problem, giving the lower bound on the slope.

$\left(W_{2}\right)$. For finite $t$, there is a bounded interval, namely $I(t)=\left[a, b+f^{\prime}\left(\kappa_{0}\right) t\right]$ such that $u_{x}=1$ outside $I(t)$, and $f$ has bounded support in $I(t)$.

$\left(W_{3}\right)$. Let $q \doteq U_{x}=u_{x}-1$. This is a conserved quantity, and satisfies the conservation law

$$
q_{t}+(f(q+1) \cdot F)_{x}=0 .
$$

By the facts that $\operatorname{sign}(q) f(q+1)=|f(q+1)|$ and $F>0$, we conclude that the $\mathbf{L}^{1}$ norm of $q$ is non-increasing in time. This leads to the BV bound on $U(t, \cdot)$.

$\left(W_{4}\right)$. By Lemma 4 , the flux $F$ is bounded away from 0 and remains bounded for finite time, and the total variation of $F$ is bounded. We consider the function $\zeta(w)$ along a characteristics $t \mapsto x(t)$ with $\dot{x}=f^{\prime}(w) F$,

$$
\zeta_{t}+f^{\prime} F \zeta_{x}=\zeta^{\prime}(w) f^{2}(w) F .
$$

Recall the definition of $\zeta$ in (1.12). We have the following

$$
\sup _{w \geq \kappa_{0}}\left|\zeta^{\prime}(w) f^{2}(w)\right| \leq C_{1}, \quad C_{1}=\max \left\{1, f^{2}\left(\kappa_{0}\right) / f^{2}(2)\right\},
$$

and

$$
\frac{d}{d w}\left(\zeta^{\prime}(w) f^{2}(w)\right) \leq C_{2} \zeta^{\prime}(w), \quad C_{2}=\max _{\kappa_{0} \leq w \leq 2}\left|2 f(w) f^{\prime}(w)\right| .
$$

The total variation of $\zeta$ can increase because of the source term in (3.14). We have

$$
\frac{d}{d t} \operatorname{TV}\{\zeta(w)\}=\operatorname{TV}\left\{\zeta^{\prime}(w) f^{2}(w) F\right\} \leq C_{1} \operatorname{TV}\{F\}+\|F\|_{\mathbf{L}^{\infty} C_{2} \operatorname{TV}\{\zeta\} .}
$$

This indicates that $\operatorname{TV}\{\zeta\}$ can grow exponentially, but remain bounded in finite time. Let $C_{3}=C_{1} \operatorname{TV}\{F\}$ and $C_{4}=C_{2}\|F\|_{\mathbf{L}^{\infty}}$, we have

$$
\operatorname{TV}\{\zeta(t)\} \leq e^{C_{4} t}\left(\operatorname{TV}\{\zeta(0)\}+\frac{C_{3}}{C_{4}}\right) .
$$




\subsection{Entropy conditions}

We introduce the entropy functions $P(u, w)$

$$
P(u, w) \doteq[u-k]_{+} \cdot[l-\zeta(w)]_{+} .
$$

Recall that the function $\zeta(\cdot)$ is defined in (1.12), which is monotone and concave. Here $(k, l) \in \Omega$ are two arbitrary constants, where

$$
\Omega \doteq\left\{(k, l): u(0, a) \leq k \leq u(T, b), \zeta\left(\kappa_{0}\right) \leq l \leq \zeta(+\infty)\right\}
$$

The operator $[\cdot]_{+}$truncates the positive part of the function, so

$$
\begin{aligned}
{[u-k]_{+} } & \doteq \begin{cases}u-k, & u>k, \\
0, & u \leq k,\end{cases} \\
{[l-\zeta(w)]_{+} } & \doteq\left\{\begin{array}{ll}
l-\zeta(w), & w<w^{l}, \\
0, & w \geq w^{l},
\end{array} \quad \text { where } \quad \zeta\left(w^{l}\right)=l .\right.
\end{aligned}
$$

Note the entropy $P$ is convex in both $w$ and $u$.

The corresponding entropy flux is $Q(u, w) F$ where $Q(u, w)$ is defined as

$$
Q(u, w) \doteq \begin{cases}{[u-k]_{+} \cdot \int_{w}^{w^{l}} f^{\prime}(s) \zeta^{\prime}(s) d s,} & w<w^{l} \\ 0, & w \geq w^{l}\end{cases}
$$

By these definitions, the following holds for all $(l, k) \in \Omega$

$$
Q_{w}(u, w)=P_{w}(u, w) \cdot f^{\prime}(w), \quad \lim _{w \rightarrow+\infty} Q(u, w)=0 .
$$

Here and in the rest of the paper we denote $P_{u}, P_{w}, Q_{u}, Q_{w}$ the partial derivatives of $P, Q$ w.r.t. $u, w$, respectively.

Next Lemma shows that the entropy is dissipative at any admissible singularity.

Lemma 6. Assume $u(t, x)$ is a weak solution of (1.1), and let u have a singularity at $(t, x)$, may it be a kink, a hyper-kink or a shock. Let $\lambda$ be its Rankine-Hugoniot speed, as in (2.7), (2.17) or (2.11). Let $\left(u^{-}, u^{+}\right)$be the left and right state for $u$, and use a similar notation for the quantities $w, F, P$ and $Q$. Then, the entropy functions $P$ are dissipative, i.e.,

$$
\lambda\left(P^{+}-P^{-}\right)-\left(Q^{+} F^{+}-Q^{-} F^{-}\right) \geq 0
$$

for all constants $(l, k) \in \Omega$, if and only if the Lax condition is satisfied

$$
f^{\prime}\left(w^{-}\right) F^{-} \geq \lambda \geq f^{\prime}\left(w^{+}\right) F^{+} .
$$


Proof. We prove the lemma for all 3 types of singularities.

Kink. At a kink, $u$ is continuous, and we have $u^{-}=u^{+}=u$ and $F^{-}=F^{+}=F$. The lemma follows from a standard argument for convex entropy. If $w^{-}<w^{+}$, we claim

$$
\lambda_{k}\left(P^{+}-P^{-}\right)-\left(Q^{+}-Q^{-}\right) F \geq 0 .
$$

Indeed, if $u<k$ or $l \leq \zeta\left(w^{-}\right)$then all terms in (3.24) are 0 , and (3.24) holds trivially. Otherwise, if $l \geq \zeta\left(w^{+}\right)$, by the convexity of the mappings $w \mapsto P$ and $w \mapsto f,(3.25)$ holds.

If $w^{-}>w^{+}$, an analogous argument leads to

$$
\lambda_{k}\left(P^{+}-P^{-}\right)-\left(Q^{+}-Q^{-}\right) F<0,
$$

for $u>k$ and $l<\zeta\left(w^{-}\right)$. This proves the Lemma for this case.

Hyper-kink. The proof for hyper-kink is completely similar. By our definitions, we have

$$
\lim _{w \rightarrow+\infty} P(u, w)=0, \quad \lim _{w \rightarrow+\infty} Q(u, w)=0,
$$

for any constants $(k, l) \in \Omega$. At a convex hyper-kink, we have $Q^{+}=P^{+}=0$, and

$$
\lambda_{h k}\left(P^{+}-P^{-}\right)-\left(Q^{+}-Q^{-}\right) F=-\lambda_{h k} P^{-}+Q^{-} F \geq 0 .
$$

But at a concave hyper-kink, we have $Q^{-}=P^{-}=0$, and

$$
\lambda_{h k}\left(P^{+}-P^{-}\right)-\left(Q^{+}-Q^{-}\right) F=\lambda_{h k} P^{+}-Q^{+} F<0,
$$

for constant $k \leq u$ and $l>\zeta\left(w^{+}\right)$. This proves that at a hyper-kink, the entropy is dissipative if and only if the hyper-kink is convex.

Shock. Across a shock, all of $u, w$ and $F$ are discontinuous. We can write

$$
\lambda_{s}\left(P^{+}-P^{-}\right)-\left(Q^{+} F^{+}-Q^{-} F^{-}\right) \doteq I_{1}+I_{2}
$$

where

$$
\begin{aligned}
& I_{1} \doteq-\lambda_{s} P^{-}+Q^{-} F^{-}, \\
& I_{2} \doteq \lambda_{s} P^{+}-Q^{+} F^{+} .
\end{aligned}
$$

The term $I_{1}$ deals with the left side of the shock. We first observe that $I_{1}=0$ if $k>u^{-}$or $l<\zeta\left(w^{-}\right)$. Now consider $k \leq u^{-}$and $l \geq \zeta\left(w^{-}\right)$. Since $\lambda_{s}<\eta_{0} F^{-}$, and by (3.26) we have

$$
I_{1}>-\eta_{0} F^{-} P^{-}+Q^{-} F^{-}>0 .
$$

This implies that the entropy is always dissipative at the left of a shock. This is not surprising, because the left of the shock is equivalent to the convex hyper-kink, which is always admissible. 
The term $I_{2}$ deals with the right side of the shock. Note that $I_{1}=0$ if $k>u^{-}$. In this case, the entropy will be dissipative across a shock if we also have $I_{2} \geq 0$.

If $k \geq u^{+}$or $l \leq \zeta\left(w^{+}\right)$, then $I_{2}=0$. Now assume $k<u^{+}$and $l>\zeta\left(w^{+}\right)$. We have

$$
I_{2}=P^{+}\left(\lambda_{s}-\frac{Q^{+}}{P^{+}} F^{+}\right)=P^{+}\left(\lambda_{s}-\frac{\int_{w^{+}}^{+\infty} f^{\prime}(s) \zeta^{\prime}(s) d s}{l-\zeta\left(w^{+}\right)} F^{+}\right) .
$$

Then, $I_{2} \geq 0$ for all $l>\zeta\left(w^{+}\right)$if and only if

$$
\lambda_{s} \geq \max _{l>\zeta\left(w^{+}\right)} \frac{\int_{w^{+}}^{+\infty} f^{\prime}(s) \zeta^{\prime}(s) d s}{l-\zeta\left(w^{+}\right)} F^{+}=f^{\prime}\left(w^{+}\right) F^{+},
$$

which is precisely the Lax condition. This completes the proof for the Lemma.

Remark 5. For smooth solutions, the entropy pairs $(P, Q F)$ satisfy the balance law

$$
P(u, w)_{t}+(Q(u, w) F)_{x}=\mathcal{S}(u, w) F,
$$

where $\mathcal{S}(u, w) F$ is the source term, with

$$
\mathcal{S}(u, w) \doteq-f(w) Q+w Q_{u}-f(w) P_{u}+f^{2}(w) P_{w} .
$$

By the definitions of $P$ and $Q$, the map $w \rightarrow \mathcal{S}$ is uniformly bounded. Indeed, we have

$$
\begin{aligned}
\lim _{w \rightarrow+\infty} f(w) Q(u, w) & =\lim _{w \rightarrow+\infty} \frac{Q(u, w)}{1 / f(w)}=\lim _{w \rightarrow+\infty} \frac{Q_{w}(u, w)}{-1 / f^{2}(w)} \\
& =[u-k]_{+} \lim _{w \rightarrow+\infty} \frac{-f^{\prime}(w) \zeta^{\prime}(w)}{-1 / f^{2}(w)}=\eta_{0}[u-k]_{+}, \\
\lim _{w \rightarrow+\infty} w Q_{u}(u, w) & \leq \lim _{w \rightarrow+\infty} \frac{\int_{w}^{+\infty} f^{\prime}(s) \zeta^{\prime}(s) d s}{1 / w} \\
& =\lim _{w \rightarrow+\infty} \frac{-f^{\prime}(w) / f^{2}(w)}{-1 / w^{2}}=\frac{1}{\eta_{0}}, \\
\lim _{w \rightarrow+\infty} f(w) P_{u} & \leq \lim _{w \rightarrow+\infty} f(w)[l-\zeta(w)]_{+}, \\
& =\lim _{w \rightarrow+\infty} \frac{\zeta(+\infty)-\zeta(w)}{1 / f(w)}=\lim _{w \rightarrow+\infty} \frac{-\zeta^{\prime}(w)}{-1 / f^{2}(w)}=1, \\
\lim _{w \rightarrow+\infty}\left|f^{2}(w) P_{w}\right| & \leq[u-k]_{+} \cdot
\end{aligned}
$$

As an immediate consequence of Lemma 6, Lax condition is equivalent to the following entropy condition: for all constants $(k, l) \in \Omega$, it holds

$$
P(u, w)_{t}+(Q(u, w) F)_{x} \leq \mathcal{S}(u, w) F,
$$


in distribution, i.e., for any smooth test function $\varphi \geq 0$ with compact support, the following holds for all $(k, l) \in \Omega$

$$
\begin{aligned}
& \int_{0}^{T} \int_{\mathbb{R}} P(u, w) \varphi_{t}+Q(u, w) F \varphi_{x} d x d t \\
& \geq \int_{\mathbb{R}}(P(u(T, x), w(T, x)) \varphi(T, x)-P(u(0, x), w(0, x)) \varphi(0, x)) d x \\
& \quad-\int_{0}^{T} \int_{\mathbb{R}} \mathcal{S}(u, w) F \varphi d x d t
\end{aligned}
$$

\section{The construction of piecewise affine approximate solutions}

The existence of entropy weak solutions for the initial value problem (1.1)-(1.2) is achieved through piecewise affine approximate solutions. In this section, we construct such an approximation. We will let $\varepsilon>0$ be the parameter for the approximation, and let $u^{\varepsilon}(t, x)$ denote the piecewise affine approximate solution.

\subsection{Piecewise affine approximation for initial data}

First, we approximate the initial date $\bar{u} \in \mathcal{W}$ by piecewise affine functions. Since $\bar{u}$ is BV on the interval $[a, b]$, by standard approximation theory, one can approximate it with piecewise affine functions that converge in $\mathbf{L}^{1}$. A possible construction is given below, which takes several steps.

Initial set of nodal points $x_{i}(0)$. The initial set of nodal points $x_{i}(0)$, $i=0, \cdots, N$ are selected through a discrete sampling of $\zeta\left(\bar{u}_{x}(x)\right)$. First, we take care of jumps in $\zeta\left(\bar{u}_{x}(x)\right)$. By property $\left(W_{4}\right)$, the function $x \mapsto \zeta\left(\bar{u}_{x}(x)\right)$ is BV. Also, outside the interval $[a, b]$ the slope $u_{x}=1$, so $\zeta\left(\bar{u}_{x}\right)$ is constant. There will be finite many points of $x_{i}(0)$ where $\zeta\left(\bar{u}_{x}\right)$ has a jump of size bigger than $\varepsilon$. All these points are selected as nodal points.

Then, every point $x_{i}(0)$ where $\bar{u}$ has a jump larger than $\varepsilon$ will also be selected. At these points we would have $\zeta\left(\bar{u}_{x}\left(x_{i}\right)\right)=\zeta(+\infty)$. In order to have a polygonal approximation to the graph of $u$, we would put 2 nodal points at jumps of $u$, i.e., $x_{i}(0)=x_{i+1}(0)$. Since $U(0, x)$ is $\mathrm{BV}, \bar{u}$ would have finitely many such jumps.

At last, we cut the interval $\left[\zeta\left(\kappa_{0}\right), \zeta(+\infty)-\varepsilon\right]$ into $N_{\zeta}$ pieces, with

$$
\zeta^{0}=\zeta\left(\kappa_{0}\right), \quad \zeta^{N_{\zeta}}=\zeta(+\infty)-\varepsilon, \quad \zeta^{j}-\zeta^{j-1} \leq \varepsilon \quad j=1, \cdots, N_{\zeta} .
$$

We need to make sure that the $\zeta^{j}=\zeta(1)$ for some $j$, i.e., the slope 1 is always sampled. Since $x \mapsto \zeta\left(\bar{u}_{x}(x)\right)$ is $\mathrm{BV}$, there are finitely many points of $x_{i}(0)$, such that $\zeta\left(\bar{u}_{x}\left(x_{i}(0)\right)\right)=\zeta^{j}$ for some $j\left(j=0, \cdots, N_{\zeta}\right)$, and

$$
\left|\zeta\left(\bar{u}_{x}\left(x_{i}\right)\right)-\zeta\left(\bar{u}_{x}\left(x_{i+1}\right)\right)\right| \leq \varepsilon,
$$


where ever $\zeta\left(\bar{u}_{x}\right)$ does not have a jump with size larger than $\varepsilon$ on the interval $\left(x_{i}, x_{i+1}\right)$.

Let's say there are totally $N$ nodal points. We would use $x_{0}(0)=a$ and $x_{N}(0)=b$ to denote the first and last nodal points, respectively.

Piecewise affine approximation to $\bar{u}(x)$. Using this set of nodal points, we can construct a piecewise affine approximation to $\bar{u}(x)$. The nodal values $u_{i}$ is set to be $u_{i}=\bar{u}\left(x_{i}\right)$ where ever $\bar{u}\left(x_{i}\right)$ is continuous, and where ever $\bar{u}$ has a shock at $x_{i}=x_{i+1}$ we set

$$
u_{i}=\bar{u}\left(x_{i}-\right), \quad u_{i+1}=\bar{u}\left(x_{i+1}+\right),
$$

Note that at a shock we have $x_{i}=x_{i+1}$ but $u_{i}<u_{i+1}$. Then, the piecewise affine approximation $u^{\varepsilon}(0, x)$ is the polygonal interpolation of $\bar{u}(x)$ through these nodal points, with vertical line at each shock $x_{i}=x_{i+1}$.

Discrete function $U^{\varepsilon}$. Using this $u^{\varepsilon}(0, x)$, we set the discrete deviation function

$$
U^{\varepsilon}(0, x) \doteq u^{\varepsilon}(0, x)-x .
$$

The nodal values are set as $U_{i}(0) \doteq U^{\varepsilon}\left(0, x_{i}\right)$ when $U^{\varepsilon}$ is continuous, and at a shock $x_{i}=x_{i+1}$,

$$
U_{i}(0) \doteq U^{\varepsilon}\left(0, x_{i}-\right), \quad U_{i+1}(0) \doteq U^{\varepsilon}\left(0, x_{i+1}+\right) .
$$

The discrete slope $w^{\varepsilon}$. The discrete slope is set to be $w^{\varepsilon}(0, x)=u_{x}^{\varepsilon}(0, x)$ where ever $u^{\varepsilon}$ is continuous, and $w^{\varepsilon}(0, x)=+\infty$ if $u^{\varepsilon}$ has a jump at $x$. Therefore, $w^{\varepsilon}$ is piecewise constant a.e., with infinite value at points where $\bar{u}$ has shocks. By using half indices, the cell value of the discrete slope on each interval $\left[x_{i}, x_{i+1}\right]$ can be computed as

$$
w_{i+\frac{1}{2}}= \begin{cases}\frac{u_{i+1}-u_{i}}{x_{i+1}-x_{i}}, & \text { if }\left[x_{i}, x_{i+1}\right] \text { is not in a shock } \\ +\infty, & \text { if }\left[x_{i}, x_{i+1}\right] \text { is in a shock }\end{cases}
$$

At the boundary we set $w_{-\frac{1}{2}}=1$ and $w_{N+\frac{1}{2}}=1$.

Discrete function $\zeta^{\varepsilon}$ and its accuracy. The discrete function $\zeta^{\varepsilon}$ computed as

$$
\zeta^{\varepsilon}(0, x) \doteq \zeta\left(u_{x}^{\varepsilon}(0, x)\right)
$$

is then a piecewise constant function. By construction, we have

$$
\left|\zeta\left(w_{i+\frac{1}{2}}\right)-\zeta\left(w_{i-\frac{1}{2}}\right)\right| \leq 2 \varepsilon,
$$

for all $i$ where $\zeta\left(\bar{u}_{x}\right)$ doesn't have a jump bigger than $\varepsilon$ at $x_{i}$. By standard approximation theory, this approximation converges in $\mathbf{L}^{1}$, and is of first order

$$
\| \zeta\left(u_{x}^{\varepsilon}(0, \cdot)-\zeta\left(\bar{u}_{x}(x)\right) \|_{\mathbf{L}^{1}} \leq C \varepsilon .\right.
$$


Furthermore, the piecewise affine approximations $u^{\varepsilon}$ ( and $U^{\varepsilon}$ ) also converge to $\bar{u}($ and $\bar{u}-x)$ in $\mathbf{L}^{1}$.

Discrete flux $F^{\varepsilon}$ and its accuracy. By using $w^{\varepsilon}$, we compute the discrete flux function

or

$$
F^{\varepsilon}\left(x ; w^{\varepsilon}\right) \doteq \exp \left\{\int_{x}^{\infty} f\left(w^{\varepsilon}(y)\right) d y\right\}
$$

$$
F^{\varepsilon}\left(u ; w^{\varepsilon}\right) \doteq \exp \left\{\int_{u}^{\infty} g\left(w^{\varepsilon}(s)\right) d s\right\}
$$

where in the second integral we use $u$ as the independent variable, as in (1.19). We will often use the simpler notations $F^{\varepsilon}(x)$ or $F^{\varepsilon}(u)$ since the dependence on $w^{\varepsilon}$ is non-local. The function $F^{\varepsilon}(x)$ will be piecewise smooth.

The nodal value $F_{i}$ are set in the following way: If $x_{i}$ is not in a shock, we set the nodal value $F_{i}=F^{\varepsilon}\left(x_{i}\right)$; If $\left[x_{i}, x_{i+1}\right]$ is a shock, then we set

$$
F_{i}=F^{\varepsilon}\left(x_{i}-\right), \quad F_{i+1}=F^{\varepsilon}\left(x_{i+1}+\right) .
$$

To ensure accuracy of the discrete flux $F^{\varepsilon}$, we assume that, (by adding more nodal points, ) for each interval $\left[x_{i}, x_{i+1}\right]$ that is not a jump,

$$
\left|f\left(w_{i+\frac{1}{2}}\right)\right|\left(x_{i+1}-x_{i}\right) \leq \varepsilon .
$$

The condition (4.2) implies

$$
e^{-\varepsilon} \leq \frac{F_{i}}{F_{i+1}} \leq e^{\varepsilon}, \quad\left|F_{i}-F_{i+1}\right| \leq \varepsilon e^{\varepsilon} F_{i}, \quad\left|F_{i}-F_{i+1}\right| \leq \varepsilon F_{i+1} .
$$

This further implies

$$
\left|U_{i+1}-U_{i}\right|=\left|\frac{w_{i+\frac{1}{2}}-1}{f\left(w_{i+\frac{1}{2}}\right)}\right| \cdot\left|f\left(w_{i+\frac{1}{2}}\right)\right|\left(x_{i+1}-x_{i}\right) \leq C \varepsilon,
$$

where $C=\max _{w \geq \kappa_{0}}|(w-1) / f(w)|$ is bounded.

Since $x \rightarrow F\left(x ; \bar{u}_{x}\right)$ is BV and uniformly bounded, there will be finitely many nodal points inserted for this purpose.

Adding more nodal points in rarefaction fans. Initial concave kinks will open up in a rarefaction fan, and need some special treatment. Assume we have a concave kink at $x_{i}$, with slopes $w_{i-\frac{1}{2}}>w_{i+\frac{1}{2}}$. We insert suitable number of nodal points at $x_{i}$, say $x_{i}=x_{j} \stackrel{\frac{1}{2}}{=} x_{j+1} \stackrel{2}{=} x_{j+2}=\cdots$, with $w_{j+\frac{1}{2}}, w_{j+1+\frac{1}{2}}, \cdots$ such that

$$
\zeta\left(w_{j+\frac{1}{2}}\right)-\zeta\left(w_{j+1+\frac{1}{2}}\right) \leq \varepsilon
$$

If the slope crosses 1, i.e. $w_{i-\frac{1}{2}}>1>w_{i+\frac{1}{2}}$, then we will select some $w_{j+\frac{1}{2}}=1$. Then, we will re-number the index $i$ accordingly. Therefore, possible large non-admissible concave kinks in the initial data will open up into a fan of small concave kinks, each of size $\leq \varepsilon$. Since $\zeta\left(\bar{u}_{x}\right)$ is BV, there 
will be finitely many rarefaction fans that need this fix, therefore the total number of added nodal points are finite.

We remark that, by Lemma 2, the condition (4.5) implies

$$
f^{\prime}\left(w_{i+1+\frac{1}{2}}\right)-f^{\prime}\left(w_{i+\frac{1}{2}}\right) \leq C \varepsilon
$$

where $C$ is the same constant as in Lemma 2. So, concave kinks are "small" kinks.

Taking care of non-admissible shocks. Finally, non-admissible shocks in the initial data will result in a rarefaction fan merging on the right of the shock. Let $\bar{u}$ have a non-admissible shock at $x_{i}=x_{i+1}$. We will insert one or multiple nodal points at $x_{i+1}$, say $x_{i+1}=x_{j}=x_{j+1}=x_{j+2}=\cdots$, with slopes $w_{j-\frac{1}{2}}, w_{j+\frac{1}{2}}, \cdots$, such that $w_{j-\frac{1}{2}}=\gamma$ where $\gamma$ follows from (2.15), and the other slopes are chosen such that (4.5) holds. Again, if the slope crosses 1 , then we will select some $w_{j+\frac{1}{2}}=1$. Of course, we will need to re-number the index $i$.

Summary. In summary, for the initial data $\bar{u}(x)$, we have now a piecewise affine approximation, represented by the nodal points $x_{i}$ and nodal values $u_{i}$. (See Figure 4.) In addition, we also have a piecewise constant function $w^{\varepsilon}$ for the slope, and a piecewise smooth function $F^{\varepsilon}$ for the flux.

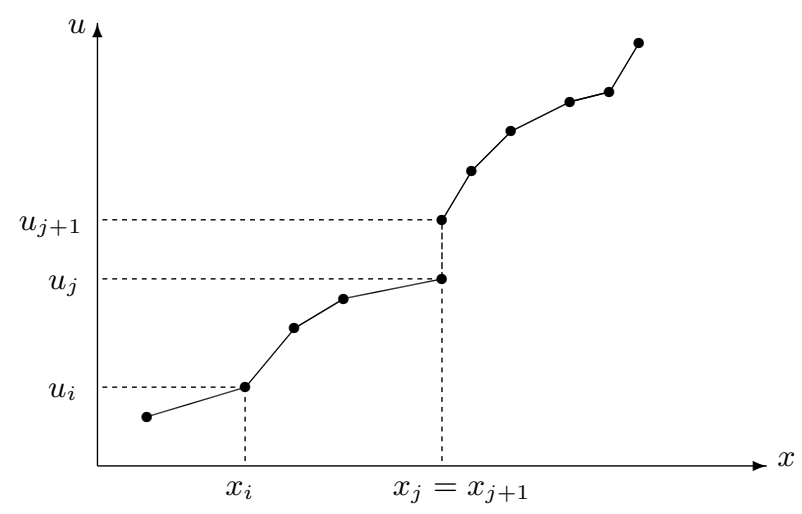

Fig. 4. Piecewise affine approximation.

\subsection{The algorithm}

The algorithm contains a set of ODEs that describes the evolutions of the nodal points $x_{i}(t)$ and nodal values $u_{i}(t)$. Each nodal point $x_{i}$ is treated as a singularity, i.e., a kink or a shock, and it travels with the corresponding speed. The evolution of the nodal value $u_{i}$ follows the characteristics from the smooth region. Details are explained below. 
If $x_{i}$ is a kink. A convex or concave kink travels with the kink speed $\lambda_{k, i}$

$$
\dot{x}_{i}(t)=\lambda_{k, i}=F_{i} \cdot \frac{f\left(w_{i+\frac{1}{2}}\right)-f\left(w_{i-\frac{1}{2}}\right)}{w_{i+\frac{1}{2}}-w_{i-\frac{1}{2}}} .
$$

The value of $u_{i}$ changes as

$$
\dot{u}_{i}(t)=\left(u_{i}\right)_{t}+\dot{x}_{i}\left(u_{i}\right)_{x}
$$

Here one can choose to use either the left or right slope,

$$
\dot{u}_{i}(t)=\lambda_{k, i} w_{i-\frac{1}{2}}-F_{i} \cdot f\left(w_{i-\frac{1}{2}}\right)=\lambda_{k, i} w_{i+\frac{1}{2}}-F_{i} \cdot f\left(w_{i+\frac{1}{2}}\right) .
$$

The second equal sign holds because $\lambda_{k, i}$ satisfies the Rankine-Hugoniot equation. Plugging (4.7) into (4.8), we get

$$
\dot{u}_{i}(t)=F_{i} \cdot \frac{w_{i-\frac{1}{2}} f\left(w_{i+\frac{1}{2}}\right)-w_{i+\frac{1}{2}} f\left(w_{i-\frac{1}{2}}\right)}{w_{i+\frac{1}{2}}-w_{i-\frac{1}{2}}} .
$$

We remark that $\dot{u}_{i}<\infty$ in (4.9). Indeed, if $w_{i+\frac{1}{2}}=w_{i-\frac{1}{2}}=w$, we have

$$
\dot{u}_{i}(t)=F_{i} \cdot\left(w f^{\prime}(w)-f(w)\right)<\infty,
$$

and if $w_{i+\frac{1}{2}} \neq w_{i-\frac{1}{2}}$, then $\dot{u}_{i}$ is also uniformly bounded because of the limits

$$
\begin{aligned}
\lim _{w \rightarrow \infty} \frac{w f\left(w_{i+\frac{1}{2}}\right)-w_{i+\frac{1}{2}} f(w)}{w_{i+\frac{1}{2}}-w} & =\lim _{w \rightarrow \infty}\left\{w_{i+\frac{1}{2}} f^{\prime}(w)-f\left(w_{i+\frac{1}{2}}\right)\right\} \\
& =w_{i+\frac{1}{2}} \eta_{0}-f\left(w_{i+\frac{1}{2}}\right)<\infty, \\
\lim _{w \rightarrow \infty} \frac{w_{i-\frac{1}{2}} f(w)-w f\left(w_{i-\frac{1}{2}}\right)}{w-w_{i-\frac{1}{2}}} & =\lim _{w \rightarrow \infty}\left\{w_{i-\frac{1}{2}} f^{\prime}(w)-f\left(w_{i-\frac{1}{2}}\right)\right\} \\
& =w_{i-\frac{1}{2}} \eta_{0}-f\left(w_{i-\frac{1}{2}}\right)<\infty .
\end{aligned}
$$

If $\left[x_{i}, x_{i+1}\right]$ is a shock. Both nodal points will travel with the same shock speed $\lambda_{s, i}$. Let $[u]_{i} \dot{=} u_{i+1}-u_{i}$ denote the size of the shock. We can write the the nodal speeds relating to the flux $F$ at the same point

$$
\dot{x}_{i}(t)=F_{i} \cdot \frac{1-\exp \left\{-\eta_{0}[u]_{i}\right\}}{[u]_{i}}, \quad \dot{x}_{i+1}(t)=F_{i+1} \cdot \frac{\exp \left\{\eta_{0}[u]_{i}\right\}-1}{[u]_{i}} .
$$

The values of $u_{i}$ and $u_{i+1}$ are updated with the slopes from the left and right (resp.)

$$
\begin{aligned}
\dot{u}_{i}(t) & =-f\left(w_{i-\frac{1}{2}}\right) F_{i}+\dot{x}_{i} w_{i-\frac{1}{2}}, \\
\dot{u}_{i+1}(t) & =-f\left(w_{i+1+\frac{1}{2}}\right) F_{i+1}+\dot{x}_{i+1} w_{i+1+\frac{1}{2}}
\end{aligned}
$$

Hyper-kinks. For any fixed $\varepsilon$, a hyper-kink at $x_{i}$ is approximated by a convex kink with a large slope $w_{i+\frac{1}{2}}$ on the right. As $\varepsilon \rightarrow 0$, we have 
$w_{i+\frac{1}{2}} \rightarrow+\infty$. Therefore, in the algorithm the hyper-kinks are treated as kinks.

The first and the last nodal point. Let $x_{0}, x_{N}$ denote the two end nodal points. Their traveling speeds are determined by the types of singularity they are connected to. Take $x_{N}$ for example, we have $w_{N+\frac{1}{2}}=1$ and $F_{N}=1$. If it is a kink, we use

$$
\dot{x}_{N}(t)=F_{N} \frac{f\left(w_{N+\frac{1}{2}}\right)-f\left(w_{N-\frac{1}{2}}\right)}{w_{N+\frac{1}{2}}-w_{N-\frac{1}{2}}}=\frac{f\left(w_{N-\frac{1}{2}}\right)}{1-w_{N-\frac{1}{2}}}, \quad \dot{u}_{N}(t)=\dot{x}_{N} .
$$

If it is connected to a shock, we use

$$
\dot{x}_{N}(t)=\frac{\exp \left\{\eta_{0}\left(u_{N}-u_{N-1}\right)\right\}-1}{u_{N}-u_{N-1}}, \quad \dot{u}_{N}(t)=\dot{x}_{N} .
$$

For $x_{0}$, it is treated in a completely similar way. If $x_{0}$ is a kink, then

$$
\dot{x}_{0}(t)=F_{0} \frac{f\left(w_{\frac{1}{2}}\right)}{w_{\frac{1}{2}}-1}, \quad \dot{u}_{0}(t)=\dot{x}_{0} .
$$

If it is connected to a shock, we use

$$
\dot{x}_{0}(t)=F_{0} \frac{1-\exp \left\{-\eta_{0}\left(u_{1}-u_{0}\right)\right\}}{u_{1}-u_{0}}, \quad \dot{u}_{0}(t)=\dot{x}_{0} .
$$

Note that in both cases, $\dot{x}_{0}(t)>0$.

Collapsing of nodal points. The nodal points $x_{i}$ are governed by a set of ODEs as in (4.7), (4.10), (4.13) or (4.14). As $t$ increases, say at $t=t^{*}$, two neighboring points $x_{i}<x_{i+1}$ might approach each other and reach the same point, such that $x_{i}\left(t^{*}\right)=x_{i+1}\left(t^{*}\right)$. There are several situations.

If $x_{i}$ and $x_{i+1}$ were both kinks before $t<t^{*}$, the collapsing can happen in two situations.

- If $w_{i+\frac{1}{2}}$ remain bounded as $t \rightarrow t^{*}$, no shock will form between $x_{i}$ and $x_{i+1}$. Then $u_{i}\left(t^{*}\right)=u_{i+1}\left(t^{*}\right)$, and the two kinks will merge into one single kink. We will remove one nodal point, and the new nodal point will then travel with the kink speed (4.7).

- If $w_{i+\frac{1}{2}}$ blows up as $t \rightarrow t^{*}$, then $\left[x_{i}, x_{i+1}\right]$ forms a shock. We will keep both nodal points, and they will travel with the shock speed, as in (4.10).

The collapsing could also happen when nearby nodal points merge into a shock. If $\left[x_{i}, x_{i+1}\right]$ is a shock, then $x_{i-1}$ could approach $x_{i}$ from the left, or $x_{i+2}$ could approach $x_{i+1}$ from the right. If this happens, we will remove the one nodal point, and re-arrange the numbering. The remaining nodal point will travel with the shock speed (4.10).

Kink changes type. It could happen that a kink changes from convex to concave and vice-versa. In this case, the algorithm remains the same, and 
the nodal point travels with kink speed. Later, we will establish the a priori bound that controls the size of concave kinks.

Treatment of the rarefaction fronts on the right of a shock. Let $x_{i}=x_{i+1}$ be a shock. It could happen that at $t$ the admissible condition (2.15) fails, and we have

$$
f^{\prime}\left(w_{i+1+\frac{1}{2}}\right) F_{i+1}-\dot{x}_{i+1} \geq \varepsilon .
$$

This will be treated in the same way as for the initial non-admissible shocks. We will insert one or more additional nodal points $x_{j}, x_{j+1}, \cdots$ between $x_{i+1}$ and $x_{i+2}$ such that $x_{j}=x_{j+1}=\cdots=x_{i+1}<x_{i+2}$, and we insert the slopes between nodes in the same way.

With this treatment, we guarantee that, at the right side of a shock,

$$
f^{\prime}\left(w_{i+1+\frac{1}{2}}\right) F_{i+1}-\dot{x}_{i+1} \leq \varepsilon,
$$

for all $t$

\section{A priori bounds for the approximate solutions}

In this section, we proof a priori estimates for the approximate solutions, requiring the additional assumption (1.21) for $f$. These estimates will be needed later on to achieve convergence of the approximate solutions, and the existence of solutions for the Cauchy problem.

For convenience we list here the properties $\left(W_{1}\right)-\left(W_{4}\right)$ that are satisfied by the discrete initial data:

$$
\begin{array}{cl}
\inf _{x} w^{\varepsilon}(0, x) \geq \kappa_{0}, & \operatorname{TV}\left\{U^{\varepsilon}(0, \cdot)\right\}<\infty, \\
\operatorname{TV}\left\{\zeta^{\varepsilon}(0, \cdot)\right\}<\infty, & x_{i}(0) \in I_{0}=[a, b] .
\end{array}
$$

Let $\left[x_{i}, x_{i+1}\right]$ be a non-shock interval and define the quantities

$$
\psi_{i+\frac{1}{2}}(t) \doteq\left|f\left(w_{i+\frac{1}{2}}(t)\right)\right| \cdot\left(x_{i+1}(t)-x_{i}(t)\right), \quad \psi(t) \doteq \sup _{i} \psi_{i+\frac{1}{2}}(t),
$$

where the supreme is taken over all $i$ where $\left[x_{i}, x_{i+1}\right]$ is not a shock. We also define

$$
\phi_{i}(t) \doteq \zeta\left(w_{i-\frac{1}{2}}(t)\right)-\zeta\left(w_{i+\frac{1}{2}}(t)\right), \quad \phi(t) \doteq \sup _{i} \phi_{i}(t),
$$

for all $i$ where $x_{i}$ is not connected to a shock.

By the construction of the initial approximate data, we have

$$
\phi(0) \leq 2 \varepsilon, \quad \psi(0) \leq \varepsilon .
$$

We have the next Lemma for the a priori estimates of the approximate solutions. 
Lemma 7. Assume that $f$ satisfies $(\boldsymbol{A})$ and (1.21). Let $u^{\varepsilon}$ be the approximate solution generated by our algorithm, with initial data $u^{\varepsilon}(0, x)$ satisfies the properties (5.1) and (5.4). Then, we have the following.

(D1). For any time $t \leq T$, we have $x \mapsto u^{\varepsilon}(t, x) \in \mathcal{W}$;

(D2). The approximate solution remains accurate for any $t \leq T$, namely

$$
\phi(t) \leq C \varepsilon, \quad \psi(t) \leq C \varepsilon .
$$

(D3). For any $t, \tau \leq T$, we have the discrete $\mathbf{L}^{1}$ continuity in time for several quantities:

$$
\begin{aligned}
\left\|u^{\varepsilon}(t, \cdot)-u^{\varepsilon}(\tau, \cdot)\right\|_{\mathbf{L}^{1}} & \leq C|t-\tau|, \\
\left\|f^{\prime}\left(u_{x}^{\varepsilon}(t, \cdot)\right)-f^{\prime}\left(u_{x}^{\varepsilon}(\tau, \cdot)\right)\right\|_{\mathbf{L}^{1}} & \leq C|t-\tau|, \\
\left\|F^{\varepsilon}\left(\cdot ; w^{\varepsilon}(t)\right)-F^{\varepsilon}\left(\cdot ; w^{\varepsilon}(\tau)\right)\right\|_{\mathbf{L}^{1}} & \leq C|t-\tau| .
\end{aligned}
$$

In all the estimates, the generic constants $C, C_{1}, C_{2}$ depend on $\kappa_{0}$, the properties of $f$, the initial total variations of $U$ and $\zeta$, but not on $\varepsilon, t, \tau$ or the points $x, y$.

The rest of this section is devoted to the proof of this Lemma.

\subsection{Property (D1)}

We first show that all the properties for $\mathcal{W}$ hold for any later time $t \leq T$. $\left(W_{1}\right)$. Lower bound on $w^{\varepsilon}$. We first derive the evolution equation for the discrete slope $w_{i+\frac{1}{2}}$. For all $w_{i+\frac{1}{2}}<\infty$, i.e., $\left[x_{i}, x_{i+1}\right]$ is not in a shock, we have

$$
\dot{w}_{i+\frac{1}{2}}(t)=\frac{\dot{u}_{i+1}-\dot{u}_{i}}{x_{i+1}-x_{i}}-w_{i+\frac{1}{2}} \frac{\dot{x}_{i+1}-\dot{x}_{i}}{x_{i+1}-x_{i}} .
$$

Using the slope on the left for $u_{i+1}$ and the slope on the right for $u_{i}$, we have

$$
\dot{u}_{i+1}=-F_{i+1} \cdot f\left(w_{i+\frac{1}{2}}\right)+\dot{x}_{i+1} w_{i+\frac{1}{2}}, \quad \dot{u}_{i}=-F_{i} \cdot f\left(w_{i+\frac{1}{2}}\right)+\dot{x}_{i} w_{i+\frac{1}{2}} .
$$

Put these back into (5.9), we get

$$
\dot{w}_{i+\frac{1}{2}}=-\frac{F_{i+1}-F_{i}}{x_{i+1}-x_{i}} \cdot f\left(w_{i+\frac{1}{2}}\right)=f^{2}\left(w_{i+\frac{1}{2}}\right) F_{i+\frac{1}{2}},
$$

where $F_{i+\frac{1}{2}}$ is some flux value between $F_{i}$ and $F_{i+1}$. We conclude that $\dot{w}_{i+\frac{1}{2}}(t) \geq 0$ for all $i$, and the lower bound on $w^{\varepsilon}(t, x)$ follows.

$\left(W_{2}\right)$. Bound on the compact support. Let $I(t)=\left[x_{0}(t), x_{N}(t)\right]$ be the interval for all the nodal points at $t$. Since all the characteristic speeds are positive, we have $\dot{x}_{0}>0$, therefore $x_{0}(t)>a$ for all $t$. For the last point $x_{N}$, since $F_{N}=1$, by the lower bound on the slope we have $\dot{x}_{N} \leq f^{\prime}\left(\kappa_{0}\right)$, so $x_{N}(t) \leq b+f^{\prime}\left(\kappa_{0}\right) t$ which is bounded for finite $t$. 
By (4.13)-(4.16), we have $\dot{u}_{0}=\dot{x}_{0}$ and $\dot{u}_{N}=\dot{x}_{N}$, we have

$$
u_{N}(t)-u_{0}(t) \leq C, \quad \forall t \in[0, T] .
$$

$\left(W_{3}\right)$. BV bound on $U^{\varepsilon}$. First we observe that no new local max or min of $U_{i}$ can form in the algorithm. This is due to the fact that $w=1$ is the equilibrium which separates the domains $w<1$ and $w>1$ into invariant regions. As a result, $U^{\prime}=0$ is an equilibrium for $U$, and $U^{\prime}$ does not change sign in time $t$ on any interval $\left[x_{i}(t), x_{i+1}(t)\right]$.

We now check how the initial local max/min values evolve in time. We discuss several cases.

Case 1. If $x_{i}$ is not in a shock. In this case, the rate of change for $U_{i}$ follows the ODE

$\dot{U}_{i}=\dot{u}_{i}-\dot{x}_{i}=\frac{F_{i}}{w_{i+\frac{1}{2}}-w_{i-\frac{1}{2}}}\left(\left(w_{i-\frac{1}{2}}-1\right) f\left(w_{i+\frac{1}{2}}\right)-\left(w_{i+\frac{1}{2}}-1\right) f\left(w_{i-\frac{1}{2}}\right)\right)$.

Case 1a. Assume that $U_{i}$ is a local maximum. This happens where $w$ crosses 1 in a rarefaction fan. By construction, since the slope $w=1$ is always selected for some interval in such a rarefaction fan, we must have another neighbor point, say $U_{i+1}$, such that $U_{i}=U_{i+1}$, so $w_{i+\frac{1}{2}}=1$ and $f\left(w_{i+\frac{1}{2}}\right)=0$. Then, by (5.12) we have $\dot{U}_{i}=\dot{U}_{i+1}=0$, and the local maximum does not change its value.

Case 1b. Assume $U_{i}$ is a local minimum, i.e., $U_{i} \leq U_{i-1}$ and $U_{i} \leq U_{i+1}$, so $w_{i-\frac{1}{2}} \leq 1 \leq w_{i+\frac{1}{2}}$. Since convex kinks are admissible, we do not have any restriction on the slopes $w_{i+\frac{1}{2}}, w_{i-\frac{1}{2}}$. By (5.12) we have

$$
\dot{U}_{i}=F_{i} \cdot\left(\left(w_{i-\frac{1}{2}}-1\right) \frac{f\left(w_{i+\frac{1}{2}}\right)-f\left(w_{i-\frac{1}{2}}\right)}{w_{i+\frac{1}{2}}-w_{i-\frac{1}{2}}}-f\left(w_{i-\frac{1}{2}}\right)\right) .
$$

By the properties of $f$ (i.e., $f(1)=0, f^{\prime}>0$ and $f^{\prime \prime}<0$ ), we have that (see Fig. 5(I))

$$
(\bar{w}-1) \frac{f(\tilde{w})-f(\bar{w})}{\tilde{w}-\bar{w}} \geq f(\bar{w}), \quad \bar{w} \leq 1 \leq \tilde{w},
$$

where the equal sign holds only if $\bar{w}=1$ or $\tilde{w}=1$. We conclude now that $\dot{U}_{i}>0$, and the local minimum will increase its value.

Case 2. If $\left[x_{i}, x_{i+1}\right]$ is in a shock. Here we need to check two situations: when $U_{i+1}$ is a local maximum, and when $U_{i}$ is a local minimum.

Case 2a. If $U_{i+1}$ is a local maximum, then we must have $w_{i+1+\frac{1}{2}} \leq 1$. If $w_{i+1+\frac{1}{2}}=1$, then $f\left(w_{i+1+\frac{1}{2}}\right)=0$ and so $\dot{U}_{i+1}=0$. Now consider $w_{i+1+\frac{1}{2}}<$ 1 and $f\left(w_{i+1+\frac{1}{2}}\right)<0$. We have

$$
\begin{aligned}
\dot{U}_{i+1}= & -f\left(w_{i+1+\frac{1}{2}}\right) F_{i+1}+\left(w_{i+1+\frac{1}{2}}-1\right) \dot{x}_{i+1} \\
= & F_{i+1}\left(-f\left(w_{i+1+\frac{1}{2}}\right)+\left(w_{i+1+\frac{1}{2}}-1\right) f^{\prime}\left(w_{i+1+\frac{1}{2}}\right)\right) \\
& +\left(1-w_{i+1+\frac{1}{2}}\right)\left(f^{\prime}\left(w_{i+1+\frac{1}{2}}\right) F_{i+1}-\dot{x}_{i+1}\right) .
\end{aligned}
$$


(I)

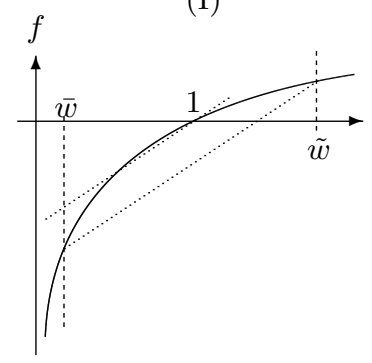

(II)

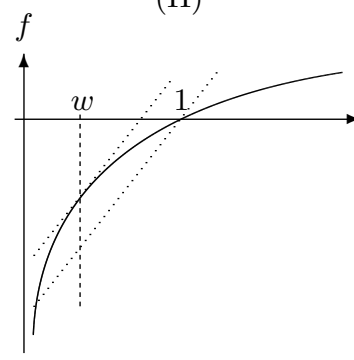

(III)

Fig. 5. Properties of the function $f$.

By the concavity of $f$, we have (see Fig. 5 , (II))

$$
-f(w)+(w-1) f^{\prime}(w)<0, \quad w<1 .
$$

The first term in (5.13) negative. The second term is bounded by (4.18), and we have

$$
\dot{U}_{i+1} \leq\left(1-w_{i+1+\frac{1}{2}}\right) \varepsilon<\varepsilon
$$

By (4.18), the size of the shock $\left(u_{i+1}-u_{i}\right)$ in this case must be sufficiently large. In fact, we must have

$$
\frac{e^{\eta_{0}\left(u_{i+1}-u_{i}\right)}-1}{u_{i+1}-u_{i}}>f^{\prime}\left(w_{i+1+\frac{1}{2}}\right)-C \varepsilon>f^{\prime}(1)-\varepsilon,
$$

which implies

$$
u_{i+1}-u_{i}>\frac{2}{\eta_{0}^{2}}\left(f^{\prime}(1)-\eta_{0}-\varepsilon\right)>\frac{2}{\eta_{0}^{2}}\left(f^{\prime}(2)-\eta_{0}\right)
$$

for $\varepsilon$ sufficiently small such that $\varepsilon \leq f^{\prime}(1)-f^{\prime}(2)$. By (5.11), the total number $N_{s}$ of such shocks is bounded

$$
N_{s}<\frac{\eta_{0}^{2}}{2} \frac{u_{N}-u_{0}}{f^{\prime}(2)-\eta_{0}}
$$

Case 2b. If $U_{i}$ is a local minimum, then we have $w_{i-\frac{1}{2}} \leq 1$ and $f\left(w_{i-\frac{1}{2}}\right) \leq 0$. By $(4.10)$ and (4.11) we get

$$
\dot{U}_{i}(t)=F_{i}\left(-f\left(w_{i-\frac{1}{2}}\right)+\left(w_{i-\frac{1}{2}}-1\right) \frac{1-\exp \left\{-\eta_{0}\left(u_{i+1}-u_{i}\right)\right\}}{u_{i+1}-u_{i}}\right) .
$$

Since any slope is admissible on the left, by the admissibility condition (2.13) with $w=1$ on the left of the shock, the following holds

$$
\frac{1-\exp \left\{-\eta_{0}\left(u_{i+1}-u_{i}\right)\right\}}{u_{i+1}-u_{i}} \leq f^{\prime}(1) \text {. }
$$


Since $w_{i-\frac{1}{2}} \leq 1$, then

$$
\dot{U}_{i}(t) \geq F_{i}\left(-f\left(w_{i-\frac{1}{2}}\right)+\left(w_{i-\frac{1}{2}}-1\right) f^{\prime}(1)\right) .
$$

By properties of $f$, we have (see Fig. 5(III))

$$
(w-1) f^{\prime}(1) \geq f(w), \quad w \leq 1 .
$$

We conclude that $\dot{U}_{i} \geq 0$, and the local minimum value is increasing.

In summary, the total variation of $U^{\varepsilon}$ could only increase in Case 2a, which occurs only at bounded number of times. Then, it holds

$$
\frac{d}{d t} \operatorname{TV}\left\{U^{\varepsilon}(t)\right\} \leq N_{s} \varepsilon
$$

We conclude that

$$
\operatorname{TV}\left\{U^{\varepsilon}(t)\right\} \leq T V\left\{U^{\varepsilon}(0)\right\}+N_{s} t \varepsilon,
$$

which remains bounded for $t \leq T$.

By Lemma 4 and (5.17), we immediately have

$$
0<F_{0} \leq F^{\varepsilon}\left(x ; w^{\varepsilon}(t)\right) \leq C, \quad \operatorname{TV}\left\{F^{\varepsilon}\left(\cdot ; w^{\varepsilon}(t)\right)\right\} \leq C .
$$

Here the values of $C, F_{0}$ depend on $\operatorname{TV}\left\{U^{\varepsilon}(0)\right\}$.

$\left(W_{4}\right)$. BV bound for $x \mapsto \zeta^{\varepsilon}$. The discrete function $\zeta^{\varepsilon}(t, x)=\zeta\left(w^{\varepsilon}(t, x)\right)$ is uniformly bounded and piecewise constant. Its evolution in time before blowup follows

$$
\dot{\zeta}\left(w_{i+\frac{1}{2}}(t)\right)=\zeta^{\prime}\left(w_{i+\frac{1}{2}}\right) \dot{w}_{i+\frac{1}{2}}=\zeta^{\prime}\left(w_{i+\frac{1}{2}}\right) f^{2}\left(w_{i+\frac{1}{2}}\right) F_{i+\frac{1}{2}} .
$$

For any $i$ where $w_{i+\frac{1}{2}}, w_{i-\frac{1}{2}}$ are finite, by using (3.15)-(3.16) we have

$$
\begin{aligned}
& \left|\dot{\zeta}\left(w_{i+\frac{1}{2}}\right)-\dot{\zeta}\left(w_{i-\frac{1}{2}}\right)\right| \\
& =\left|\zeta^{\prime}\left(w_{i+\frac{1}{2}}\right) f^{2}\left(w_{i+\frac{1}{2}}\right) F_{i+\frac{1}{2}}-\zeta^{\prime}\left(w_{i-\frac{1}{2}}\right) f^{2}\left(w_{i-\frac{1}{2}}\right) F_{i-\frac{1}{2}}\right| \\
& \leq C_{1}\left\|F^{\varepsilon}\right\|_{\mathbf{L} \infty}\left|\zeta\left(w_{i+\frac{1}{2}}\right)-\zeta\left(w_{i-\frac{1}{2}}\right)\right|+C_{2}\left|F_{i+\frac{1}{2}}-F_{i-\frac{1}{2}}\right| .
\end{aligned}
$$

If $\left[x_{i}, x_{i+1}\right]$ is a shock so $w_{i+\frac{1}{2}}=+\infty$, then we have

$$
\frac{d}{d t}\left|\zeta\left(w_{i+1+\frac{1}{2}}\right)-\zeta(+\infty)\right|+\frac{d}{d t}\left|\zeta(+\infty)-\zeta\left(w_{i-\frac{1}{2}}\right)\right| \leq 0,
$$

since $\zeta\left(w_{i+\frac{1}{2}}\right)$ is non-decreasing (because $w_{i+\frac{1}{2}}$ is non-decreasing and $\zeta$ is a monotone increasing function). Summing over all cases, we conclude, using $C_{3}, C_{4}$ for the constants,

$$
\frac{d}{d t} \operatorname{TV}\left\{\zeta^{\varepsilon}\right\} \leq C_{3}+C_{4} \operatorname{TV}\left\{\zeta^{\varepsilon}\right\}
$$

By a standard comparison argument, $\operatorname{TV}\left\{\zeta^{\varepsilon}\right\}$ can grow exponentially, but remain bounded for finite time,

$$
\operatorname{TV}\left\{\zeta^{\varepsilon}(t)\right\} \leq \exp \left\{C_{4} t\right\}\left(\operatorname{TV}\left\{\zeta^{\varepsilon}(0\}+\frac{C_{3}}{C_{4}}\right)\right.
$$




\subsection{Property (D2): Accuracy of the approximation}

We recall the definitions (5.2) for $\psi$. We first observe that during interaction $\psi$ does not change, Now consider a time $t$ with no interactions. Consider a non-shock interval $\left[x_{i}, x_{i+1}\right]$. We have

$$
\begin{aligned}
& \dot{\psi}_{i+\frac{1}{2}}=\operatorname{sign}\left(w_{i+\frac{1}{2}}-1\right) f^{\prime}\left(w_{i+\frac{1}{2}}\right) \dot{w}_{i+\frac{1}{2}}\left(x_{i+1}-x_{i}\right)+\left|f\left(w_{i+\frac{1}{2}}\right)\right| \cdot\left(\dot{x}_{i+1}-\dot{x}_{i}\right) \\
& =-f^{\prime}\left(w_{i+\frac{1}{2}}\right) \cdot\left|f\left(w_{i+\frac{1}{2}}\right)\right| \cdot\left(F_{i+1}-F_{i}\right)+\left|f\left(w_{i+\frac{1}{2}}\right)\right| \cdot\left(\dot{x}_{i+1}-\dot{x}_{i}\right) \\
& =\left|f\left(w_{i+\frac{1}{2}}\right)\right|\left(\dot{x}_{i+1}-f^{\prime}\left(w_{i+\frac{1}{2}}\right) F_{i+1}\right)+\left|f\left(w_{i+\frac{1}{2}}\right)\right|\left(f^{\prime}\left(w_{i+\frac{1}{2}}\right) F_{i}-\dot{x}_{i}\right) \cdot(5.23)
\end{aligned}
$$

Note that $f^{\prime}\left(w_{i+\frac{1}{2}}\right) F_{i}$ and $f^{\prime}\left(w_{i+\frac{1}{2}}\right) F_{i+1}$ are characteristic speeds at $x_{i}, x_{i+1}$ from within the interval. The two terms in (5.23) could be positive only if the nodal points $x_{i}$ and $x_{i+1}$ are in a rarefaction fan, with concave kinks, where (4.5) must hold. If the slope $w_{i+\frac{1}{2}}$ is bounded, say $w_{i+\frac{1}{2}} \leq 5$, so no blowup occurs, then we can use the estimates (by Lemma 2)

$$
\begin{gathered}
f^{\prime}\left(w_{i+\frac{1}{2}}\right) F_{i}-\dot{x}_{i} \leq F_{i}\left(f^{\prime}\left(w_{i+\frac{1}{2}}\right)-f^{\prime}\left(w_{i-\frac{1}{2}}\right)\right) \leq C F_{i} \phi, \\
\dot{x}_{i+1}-f^{\prime}\left(w_{i+\frac{1}{2}}\right) F_{i+1} \leq F_{i+1}\left(f^{\prime}\left(w_{i+1+\frac{1}{2}}\right)-f^{\prime}\left(w_{i+\frac{1}{2}}\right)\right) \leq C F_{i+1} \phi,
\end{gathered}
$$

where the constant $C$ is as in Lemma 2, and it depends on $\operatorname{TV}\left\{U^{\varepsilon}(0)\right\}$. We have, for all $i$

$$
\dot{\psi}_{i+\frac{1}{2}} \leq C_{1} \phi, \quad C_{1}=2 C\left\|F^{\varepsilon}\right\|_{\mathbf{L} \infty} \cdot \max _{\kappa_{0} \leq w \leq 5}|f(w)| .
$$

If blowup occurs, then we need more detailed analysis. Now consider $w_{i+\frac{1}{2}}$ very large, say $w_{i+\frac{1}{2}}>5$. We have

$$
\begin{aligned}
I_{1} & \doteq f\left(w_{i+\frac{1}{2}}\right)\left(\dot{x}_{i+1}-f^{\prime}\left(w_{i+\frac{1}{2}}\right) F_{i+1}\right) \\
& =f\left(w_{i+\frac{1}{2}}\right)\left(\frac{f\left(w_{i+1+\frac{1}{2}}\right)-f\left(w_{i+\frac{1}{2}}\right)}{w_{i+1+\frac{1}{2}}-w_{i+\frac{1}{2}}}-f^{\prime}\left(w_{i+\frac{1}{2}}\right)\right) \cdot F_{i+1} .
\end{aligned}
$$

Since $w_{i+\frac{1}{2}} \geq w_{i+1+\frac{1}{2}}$, so $w_{i+\frac{1}{2}}$ would blowup first. Applying (3.9) in Lemma 3 , with $w=w_{i+\frac{1}{2}}$ and $\bar{w}=w_{i+1+\frac{1}{2}}$, we get the following estimate for $I_{1}$,

$$
I_{1} \leq C F_{i+1}\left(\zeta\left(w_{i+\frac{1}{2}}\right)-\zeta\left(w_{i+1+\frac{1}{2}}\right)\right)=C F_{i+1} \phi_{i+1} \leq C_{2} \phi
$$

Here the constant $C$ is as in (3.8) in Lemma 3, and $C_{2}=C\left\|F^{\varepsilon}\right\|_{\mathbf{L}^{\infty}}$. For the second term in (5.23), we observe

$$
\begin{aligned}
I_{2} & \doteq f\left(w_{i+\frac{1}{2}}\right)\left(f^{\prime}\left(w_{i+\frac{1}{2}}\right) F_{i}-\dot{x}_{i}\right) \\
& =f\left(w_{i+\frac{1}{2}}\right)\left(f^{\prime}\left(w_{i+\frac{1}{2}}\right)-\frac{f\left(w_{i-\frac{1}{2}}\right)-f\left(w_{i+\frac{1}{2}}\right)}{w_{i-\frac{1}{2}}-w_{i+\frac{1}{2}}}\right) \cdot F_{i}
\end{aligned}
$$


Since $w_{i-\frac{1}{2}} \geq w_{i+\frac{1}{2}}$, so $w_{i-\frac{1}{2}}$ would blow up first. We can apply (3.8) in Lemma 3 , with $\bar{w} \stackrel{\frac{1}{2}}{=} w_{i+\frac{1}{2}}$ and $w=w_{i-\frac{1}{2}}$, and get

$$
I_{2} \leq C F_{i}\left(\zeta\left(w_{i+\frac{1}{2}}\right)-\zeta\left(w_{i-\frac{1}{2}}\right)\right)=C F_{i} \phi_{i} \leq C_{3} \phi
$$

Here the constant $C$ is as in (3.9) and $C_{3}=C\left\|F^{\varepsilon}\right\|_{\mathbf{L}^{\infty}}$. Combining (5.24), (5.25) and (5.26), choosing some constant $C=\max \left\{C_{1}, C_{2}+C_{3}\right\}$, we obtain

$$
\dot{\psi}_{i+\frac{1}{2}} \leq C \phi
$$

For the evolution of $\phi$, we recall the definitions in (5.3), and notice that $\phi$ measures the maximum size of non-admissible (concave) kinks. We observe that during interaction $\phi$ is decreasing. Indeed, two nearby concave kinks can not approach each other. Therefore, a concave kink could only interact with either a convex kink or a shock, where it would be cancelled, causing a decreasing effect on $\phi$.

Since $\zeta$ is an increasing function, we will have $\phi>0$ only in a rarefaction wave with concave kinks. Let's consider a concave kink at $x_{i}(t)$, with slope $\infty>w_{i-\frac{1}{2}}(t)>w_{i+\frac{1}{2}}(t)$. By using (3.15)-(3.16), we have

$$
\begin{aligned}
\dot{\phi}_{i} & =\dot{\zeta}\left(w_{i+\frac{1}{2}}\right)-\dot{\zeta}\left(w_{i-\frac{1}{2}}\right) \\
& =\zeta^{\prime}\left(w_{i+\frac{1}{2}}\right) f^{2}\left(w_{i+\frac{1}{2}}\right) F_{i+\frac{1}{2}}-\zeta^{\prime}\left(w_{i-\frac{1}{2}}\right) f^{2}\left(w_{i-\frac{1}{2}}\right) F_{i-\frac{1}{2}} \\
& \leq C_{1}\left\|F^{\varepsilon}\right\|_{\mathbf{L} \infty}\left(\zeta\left(w_{i+\frac{1}{2}}\right)-\zeta\left(w_{i-\frac{1}{2}}\right)\right)+C_{2}\left|F_{i+\frac{1}{2}}-F_{i-\frac{1}{2}}\right| \\
& \leq C_{3} \phi+C_{4} \psi
\end{aligned}
$$

Here we used

$$
\left|F_{i+\frac{1}{2}}-F_{i-\frac{1}{2}}\right| \leq\left|F_{i-1}-F_{i}\right|+\left|F_{i}-F_{i+1}\right|
$$

and

$$
\left|F_{i+1}-F_{i}\right| \leq F_{i}\left|1-\exp \left\{-\psi_{i+\frac{1}{2}}\right\}\right| \leq \psi F_{i}, \quad\left|F_{i}-F_{i-1}\right| \leq \psi F_{i-1},
$$

and the constants $C_{3}, C_{4}$ depend on $C_{1}, C_{2}$ in (3.15)-(3.16) and $\left\|F^{\varepsilon}\right\|_{\mathbf{L}}$.

Taking supreme over $i$ in (5.27) and (5.28), we get

$$
\dot{\psi} \leq C \phi, \quad \dot{\phi} \leq C_{3} \psi+C_{4} \phi
$$

Using a standard comparison argument, we conclude that, if $\psi(0), \phi(0) \leq 2 \varepsilon$ for some $\varepsilon$ sufficiently small, then

$$
\phi(t) \leq C \varepsilon, \quad \psi(t) \leq C \varepsilon
$$

where $C$ does not depend on $\varepsilon$. 


\subsection{Property (D3): Discrete $\mathbf{L}^{1}$ Continuity in time.}

Here we discuss the $\mathbf{L}^{1}$ continuity in time for $u^{\varepsilon}, f^{\prime}\left(w^{\varepsilon}\right)$ and the flux $F^{\varepsilon}$.

For $u^{\varepsilon}$ : Since $u^{\varepsilon}$ remains unchanged outside the bounded interval $I(t)=$ $\left[x_{0}(t), x_{N}(t)\right]$, and $u^{\varepsilon}$ is $\mathrm{BV}$ on $I(t)$, it suffices to show that $u_{t}^{\varepsilon}$ is absolutely integrable in $x$. To understand how $u^{\varepsilon}$ changes in time for fixed $x$, we consider a point $(t, x)$ and $x \in\left[x_{i}, x_{i+1}\right)$. We have

$$
u^{\varepsilon}(t, x)=u_{i}(t)+\left(x-x_{i}(t)\right) \cdot w_{i+\frac{1}{2}}(t) .
$$

Then

$$
\begin{aligned}
u_{t}^{\varepsilon} & =\dot{u}_{i}+\left(x-x_{i}\right) \dot{w}_{i+\frac{1}{2}}-\dot{x}_{i} w_{i+\frac{1}{2}} \\
& =\left(-F_{i} f\left(w_{i+\frac{1}{2}}\right)+\dot{x}_{i} w_{i+\frac{1}{2}}\right)-\left(x-x_{i}\right) \frac{F_{i+1}-F_{i}}{x_{i+1}-x_{i}} f\left(w_{i+\frac{1}{2}}\right)-\dot{x}_{i} w_{i+\frac{1}{2}} \\
& =-f\left(w_{i+\frac{1}{2}}\right)\left(F_{i}+\left(x-x_{i}\right) \frac{F_{i+1}-F_{i}}{x_{i+1}-x_{i}}\right)
\end{aligned}
$$

Therefore

$$
u_{t}^{\varepsilon}=-f\left(u_{x}^{\varepsilon}\right) \widehat{F}^{\varepsilon},
$$

where $\widehat{F}^{\varepsilon}$ is a linear interpolation of the discrete flux $F^{\varepsilon}$ through nodal points.

$$
\widehat{F}^{\varepsilon}\left(x ; w^{\varepsilon}(t)\right) \doteq F_{i}+\left(x-x_{i}\right) \frac{F_{i+1}-F_{i}}{x_{i+1}-x_{i}}, \quad x \in\left[x_{i}(t), x_{i+1}(t)\right] .
$$

We see that $u_{t}^{\varepsilon}$ is absolutely integrable because $\widehat{F}^{\varepsilon}$ is uniformly bounded and $f\left(u_{x}^{\varepsilon}\right)$ is absolutely integrable (because $u^{\varepsilon} \in \mathcal{W}$ so we can use (3.10) in Lemma 4).

For $f^{\prime}\left(w^{\varepsilon}\right)$ : Since $f^{\prime}=f^{\prime}(1)$ outside $I(t)$, and $f^{\prime}$ is BV, it suffices to show that $\frac{d}{d t} f^{\prime}\left(w_{i+\frac{1}{2}}(t)\right)$ is uniformly bounded. Using the decay property (3.4), we have

$$
\frac{d}{d t} f^{\prime}\left(w_{i+\frac{1}{2}}(t)\right)=f^{\prime \prime}\left(w_{i+\frac{1}{2}}\right) \dot{w}_{i+\frac{1}{2}}=f^{\prime \prime}\left(w_{i+\frac{1}{2}}\right) f^{2}\left(w_{i+\frac{1}{2}}\right) F_{i+\frac{1}{2}}<\infty .
$$

For $F^{\varepsilon}$ : Since the flux $F^{\varepsilon}$ is non-local, the continuity in time is not so trivial. Let $(t, x)$ be a point such that $x \in\left[x_{i}, x_{i+1}\right)$ for some $i$ and write $u^{\varepsilon}=u^{\varepsilon}(t, x)$. By using $u^{\varepsilon}$ as the integration variable, we can express the flux as

$$
\begin{aligned}
F^{\varepsilon}\left(u^{\varepsilon} ; w^{\varepsilon}\right) & =\exp \int_{u^{\varepsilon}}^{\infty} g\left(u_{x}^{\varepsilon}(t, s)\right) d s \\
& =\exp \left\{g\left(w_{i+\frac{1}{2}}\right)\left(u_{i+1}-u^{\varepsilon}\right)+\sum_{j \geq i+1}^{N} g\left(w_{j+\frac{1}{2}}\right)\left(u_{j+1}-u_{j}\right)\right\} .
\end{aligned}
$$


So, by summation-by-parts, we have

$$
\begin{aligned}
F_{t}^{\varepsilon}= & F^{\varepsilon} \cdot\left\{g^{\prime}\left(w_{i+\frac{1}{2}}\right) \dot{w}_{i+\frac{1}{2}}\left(u_{i+1}-u^{\varepsilon}\right)+g\left(w_{i+\frac{1}{2}}\right)\left(\dot{u}_{i+1}-u_{t}^{\varepsilon}\right)\right\} \\
& +F^{\varepsilon} \cdot\left\{\sum_{j \geq i+1}^{N}\left(g^{\prime}\left(w_{j+\frac{1}{2}}\right) \dot{w}_{j+\frac{1}{2}}\left(u_{j+1}-u_{j}\right)+g\left(w_{j+\frac{1}{2}}\right)\left(\dot{u}_{j+1}-\dot{u}_{j}\right)\right)\right\} \\
\doteq & R_{1}+R_{2}+R_{3}
\end{aligned}
$$

where $R_{1}, R_{2}, R_{3}$ are the corresponding 3 terms

$$
\begin{aligned}
& R_{1} \doteq F^{\varepsilon} \cdot g^{\prime}\left(w_{i+\frac{1}{2}}\right) f^{2}\left(w_{i+\frac{1}{2}}\right) F_{i+\frac{1}{2}}\left(u_{i+1}-u^{\varepsilon}\right)-F^{\varepsilon} \cdot g\left(w_{i+\frac{1}{2}}\right) u_{t}^{\varepsilon} \\
& R_{2} \doteq F^{\varepsilon} \cdot \sum_{j \geq i+1}^{N}\left(g^{\prime}\left(w_{j+\frac{1}{2}}\right) f^{2}\left(w_{j+\frac{1}{2}}\right) F_{j+\frac{1}{2}}\left(u_{j+1}-u_{j}\right)\right) \\
& R_{3} \doteq F^{\varepsilon} \cdot \sum_{j \geq i+1}^{N} \dot{u}_{j}\left(g\left(w_{j+\frac{1}{2}}\right)-g\left(w_{j-\frac{1}{2}}\right)\right) .
\end{aligned}
$$

Now we discuss all 3 terms. For $R_{1}$, we have that

$$
\lim _{w \rightarrow \infty} g^{\prime}(w) f^{2}(w)=\lim _{w \rightarrow \infty}\left(w f^{\prime}(w)-f(w)\right) \frac{f^{2}(w)}{w^{2}}<\infty,
$$

thanks to Lemma 1. Furthermore, $F^{\varepsilon}$ and $g\left(w_{i+\frac{1}{2}}\right)$ are uniformly bounded, and $u_{t}^{\varepsilon}$ is computed in (5.30) and is absolutely integrable. Therefore, $R_{1}$ is absolutely integrable.

For $R_{2}$, because of (5.32) and that $u^{\varepsilon}$ is $\mathrm{BV}$ on the bounded interval $[a(t), b(t)]$, so $R_{2}$ is bounded.

For $R_{3}$, there are various cases. If $x_{j}$ is a kink, from (4.9) we see $\dot{u}_{j}$ is bounded. Since $x \mapsto g\left(u_{x}^{\varepsilon}\right)$ is BV (by Lemma 4 ), then the sum over all kinks is bounded. If $x_{j}$ is on the left of a shock so that $w_{i+\frac{1}{2}}=+\infty$, then we use (4.11) for $\dot{u}_{i}$. We have

$$
\begin{aligned}
& \dot{u}_{i}\left(g\left(w_{i-\frac{1}{2}}\right)-\eta_{0}\right) \\
& =F_{i}\left(\frac{1-\exp \left\{-\eta_{0}[u]_{i}\right\}}{[u]_{i}} w_{i-\frac{1}{2}}-f\left(w_{i-\frac{1}{2}}\right)\right)\left(g\left(w_{i-\frac{1}{2}}\right)-\eta_{0}\right) \\
& =F_{i}\left(\left(\frac{1-\exp \left\{-\eta_{0}[u]_{i}\right\}}{[u]_{i}}-\eta_{0}\right)+\left(\eta_{0}-g\left(w_{i-\frac{1}{2}}\right)\right)\right)\left(f\left(w_{i-\frac{1}{2}}\right)-\eta_{0} w_{i-\frac{1}{2}}\right) .
\end{aligned}
$$

By Lemma 1, $f\left(w_{i-\frac{1}{2}}\right)-\eta_{0} w_{i-\frac{1}{2}}$ is uniformly bounded. Also, since $g\left(u_{x}^{\varepsilon}\right)$ is $\mathrm{BV}$, then the sum of $\left|\eta_{0}-g\left(w_{i-\frac{1}{2}}\right)\right|$ over the left point of shocks is bounded. Furthermore, we have

$$
\sum_{i}\left|\frac{1-\exp \left\{-\eta_{0}[u]_{i}\right\}}{[u]_{i}}-\eta_{0}\right| \leq \sum_{i} \frac{1}{2} \eta_{0}^{2}[u]_{i} \leq \frac{1}{2} \eta_{0}^{2}\left(u_{N}-u_{i}\right) .
$$

where the summation is taking over all shocks. Therefore, the sum over all left point of the shocks in $R_{3}$ is bounded. Finally, if $x_{j}$ is on the right of a 
shock, it is completely similar. We omit the details. Summing over all cases, we conclude that $R_{3}$ is uniformly bounded.

In conclusion, for any $t \leq T$, the derivative $F_{t}^{\varepsilon}$ is absolutely integrable in $x$, proving the $\mathbf{L}^{1}$ continuity in time for $F^{\varepsilon}$.

This completes the proof of Lemma 7 .

\subsection{Well-posedness of the algorithm}

The well-posedness of the algorithm follows from the following facts.

1. For any given $\varepsilon$, the total number of nodal points $x_{i}$ remains bounded for all time. Indeed, we have the following.

- At every interaction nodal points can only merge, causing a decrease in the number of nodal points.

- The total nodal points could increase when we insert an additional nodal point at the right of a shock when the admissible condition (2.15) fails. Due to the fact that all slopes $w_{i+\frac{1}{2}}$ increases in time, and $U^{\varepsilon}$ and $\zeta^{\varepsilon}$ have bounded variations, the largest possible number of inserted nodal points are bounded, of order $\mathcal{O}(1 / \varepsilon)$.

2. Then, total number of interactions are bounded for $t \leq T$. Indeed, at any interaction waves merge. At the right side of a shock, if additional nodes are added at $t$, due to the continuity of the global flux and shock strength, the total number of time this node enters and leaves the shock is bounded. Thus, total number of interactions are bounded for $t \leq T$.

3 . The traveling speed of a kink, determined by (4.7) is uniformly bounded for all time $t \leq T$. Furthermore, outside times of interactions, the speed is Lipschitz continuous in time. Therefore we have existence and uniqueness of these ODEs for finite time.

4. For any kink at $x_{i}$, the ODEs for $u_{i}$ as in (4.9), is uniformly bounded and Lipschitz continuous in time. This give well-posedness of these ODEs.

5. If $\left[x_{i}, x_{i+1}\right]$ is a shock, then the speed $\dot{x}_{i}=\dot{x}_{i+1}$, determined by (4.10), is uniformly bounded and Lipschitz for finite shock size $[u]_{i}$. Therefore, these ODEs are well-posed for finite time.

6. The only ODEs remained in question are the ones for $u_{i}, u_{i+1}$ where $\left[x_{i}, x_{i+1}\right]$ is a shock. In this case, $\dot{u}_{i}$ and $\dot{u}_{i+1}$ will be computed as in (4.11) and (4.12). When the slope $w$ does not blow up, these are Lipschitz and uniformly bounded. In case of blowup, we observe that, by the a priori lower bound on the slope, we have, for any time $t$,

$$
u_{i}(t) \leq u_{i+1}(t), \quad \forall i
$$

Therefore, if the slope blows up along a characteristic $x_{i}(t)$ at $t=\tilde{t}$, we must have

$$
\int_{0}^{\tilde{t}} \dot{u}_{i}(t) d t<\infty
$$

yielding well-posedness of these ODEs. 


\section{Convergence of the approximate solutions - Existence of entropy weak solutions}

In this section, we first establish the convergence of the approximate solutions, obtaining the existence of BV weak solutions with the additional assumption (1.21). Then, we derive the discrete entropy inequality. Through the convergence of these inequalities we obtain the existence of entropy weak solutions. Finally, we drop the assumption (1.21), proving the main Theorem 1 .

\subsection{Existence of weak solutions}

From (5.30) we see that $u^{\varepsilon}(t, x)$ solves the differential equation

$$
u_{t}^{\varepsilon}+f\left(u_{x}^{\varepsilon}\right) \widehat{F}^{\varepsilon}=0,
$$

where $\widehat{F}^{\varepsilon}$, defined in $(5.31)$, is a linear interpolation of the discrete flux $F^{\varepsilon}$ through nodal points. We write (6.1) in conservative form

$$
u_{t}^{\varepsilon}-F_{x}^{\varepsilon}=e^{\varepsilon}, \quad e^{\varepsilon}(t, x) \doteq-F_{x}^{\varepsilon}-f\left(u_{x}^{\varepsilon}\right) \widehat{F}^{\varepsilon},
$$

where $e^{\varepsilon}$ is the error term. Since all the singularities (kinks and shocks) travel with a speed that satisfies the Rankine-Hugoniot condition, our approximate solution $u^{\varepsilon}$ satisfies the following integral form of the equation. For every test function $\varphi \in \mathcal{C}^{\infty}$ with compact support, we have

$$
\begin{gathered}
\int_{0}^{T} \int_{\mathbb{R}}\left(u^{\varepsilon} \varphi_{t}-F^{\varepsilon} \varphi_{x}\right) d x d t=\int_{\mathbb{R}}\left(u^{\varepsilon}(T, x) \varphi(T, x)-u^{\varepsilon}(0, x) \varphi(0, x)\right) d x \\
-\int_{0}^{T} \int_{\mathbb{R}} e^{\varepsilon}(t, x) \varphi d x d t
\end{gathered}
$$

To achieve the existence of weak solutions, we claim that we can extract a subsequence $\varepsilon \rightarrow 0$ such that, for some limit functions $u(t, x)$ and $F(t, x)$, one has

$(\mathrm{c} 1) u^{\varepsilon}(0, \cdot) \rightarrow u(0, \cdot)$ and $u^{\varepsilon}(T, \cdot) \rightarrow u(T, \cdot)$ in $\mathbf{L}_{l o c}^{1}(\mathbb{R})$;

(c2) $u^{\varepsilon}(t, x) \rightarrow u(t, x)$ and $F^{\varepsilon}\left(x ; w^{\varepsilon}(t)\right) \rightarrow F(t, x)$ in $\mathbf{L}_{l o c}^{1}([0, T] \times \mathbb{R})$;

(c3) for any given $a<b$,

$$
\int_{a}^{b}\left|e^{\varepsilon}(t, x)\right| d x \rightarrow 0, \quad \text { uniformly for } t \in[0, T]
$$

(c4) the identity

$$
F(t, x)=\exp \left\{\int_{x}^{\infty} f\left(u_{x}(t, y)\right) d y\right\}
$$

holds for a.e. $(t, x) \in[0, T] \times \mathbb{R}$. 
This will imply that $u$ is a weak solution, with the correct flux $F$. Now we will prove these claims.

(c1). As $x$ ranges on any bounded interval $[a, b]$, the functions $u^{\varepsilon}(t, \cdot)$ are uniformly bounded, and monotone increasing w.r.t. $x$. Therefore, by Helly's compactness theorem, there exists a subsequence $\varepsilon_{k} \rightarrow 0$ such that the corresponding functions $u^{\varepsilon_{k}}(0, \cdot)$ and $u^{\varepsilon_{k}}(T, \cdot)$ converge pointwise everywhere, hence also in $\mathbf{L}_{l o c}^{1}(\mathbb{R})$. This proves $(\mathrm{c} 1)$.

(c2). Our approximate solutions $u^{\varepsilon}$ satisfies the followings.

- As $x$ ranges on any fixed interval $[a, b]$, the total variation of the maps $x \mapsto u^{\varepsilon}(t, x)$ and $x \mapsto F^{\varepsilon}\left(x ; w^{\varepsilon}(t)\right)$ remains bounded, uniformly w.r.t. $t$ and $\varepsilon$;

- The maps $t \mapsto u^{\varepsilon}(t, \cdot)$ and $t \mapsto F^{\varepsilon}\left(\cdot ; w^{\varepsilon}(t, \cdot)\right)$ are uniformly Lipschitz continuos from $[0, T]$ into $\mathbf{L}^{1}([a, b])$.

Using a standard compactness argument (see for example Theorem 2.4 in [6]), we get (c2).

(c3). For $x \in\left[x_{i}, x_{i+1}\right]$ that is not a shock, the error is

$$
e^{\varepsilon}(t, x)=f\left(w_{i+\frac{1}{2}}\right) F^{\varepsilon}(x)-f\left(w_{i+\frac{1}{2}}\right) \widehat{F}^{\varepsilon}(x)=f\left(w_{i+\frac{1}{2}}\right)\left(F^{\varepsilon}(x)-\widehat{F}^{\varepsilon}(x)\right) .
$$

Since $F^{\varepsilon}$ is smooth on $\left[x_{i}, x_{i+1}\right]$, the linear interpolation error $\widehat{F}^{\varepsilon}$ satisfies the standard estimate

$$
\begin{aligned}
\left|F^{\varepsilon}(x)-\widehat{F}^{\varepsilon}(x)\right| & \leq \frac{1}{2}\left(x_{i+1}-x_{i}\right)^{2} \max \left|F_{x x}^{\varepsilon}\right| \\
& \leq\left(x_{i+1}-x_{i}\right)^{2} f^{2}\left(w_{i+\frac{1}{2}}\right) \max \left\{F^{\varepsilon}\right\} \leq C \varepsilon^{2}
\end{aligned}
$$

where the constant $C$ depends on $\left\|F^{\varepsilon}\right\|_{\mathbf{L}^{\infty}}$. Therefore, we have

$$
\left|e^{\varepsilon}(t, x)\right| \leq C\left|f\left(u_{x}^{\varepsilon}\right)\right| \cdot \varepsilon^{2} .
$$

According to (3.10), the integral of the function $x \mapsto\left|f\left(u_{x}^{\varepsilon}(t, x)\right)\right|$ remains uniformly bounded, uniformly in $t \in[0, T]$. This proves (c3).

(c4). To show that $F^{\varepsilon}$ converges to the correct limit, we make a variable change, and use $(t, u)$ as the independent variables instead of $(t, x)$. This will take several steps.

Step 1. Considering $x$ as a function of $(t, u)$, our algorithm generates a piecewise affine approximation $x^{\varepsilon}(t, u)$. Thanks to the lower bound on the slope $w^{\varepsilon} \geq \kappa_{0}>0$, the map $u \mapsto x^{\varepsilon}(t, u)$ is uniformly Lipschitz continuous with Lipschitz constant $1 / \kappa_{0}$.

Step 2. The derivative $x_{u}^{\varepsilon}(t, u)$ is non-negative and uniformly bounded. We claim that the map $u \mapsto x_{u}^{\varepsilon}(t, u)$ is BV. Indeed, by construction $x_{u}^{\varepsilon}=1$ outside the bounded interval $I_{u}(t)$. We also have $\max \left\{x_{u}^{\varepsilon}\right\}=1 / \kappa_{0}$, and $x_{u}^{\varepsilon}=0$ at places where $u^{\varepsilon}$ has shocks. Define the function

$$
\mathcal{H}(s) \doteq \zeta\left(\frac{1}{s}\right) .
$$


Then, $u \mapsto \mathcal{H}\left(x_{u}^{\varepsilon}(t, u)\right)$ is BV because $x \mapsto \zeta\left(w^{\varepsilon}(t, x)\right)$ is BV. Furthermore, the derivative of $\mathcal{H}$ is

$$
\mathcal{H}^{\prime}(s)=-\frac{1}{s^{2}} \zeta^{\prime}\left(\frac{1}{s}\right)= \begin{cases}\frac{1}{s^{2}} \frac{1}{f^{2}(2)}, & \kappa_{0} \leq \frac{1}{s} \leq 2, \\ \frac{1}{s^{2}} \frac{1}{f^{2}(1 / s)}, & \frac{1}{s}>2 .\end{cases}
$$

This is strictly positive and uniformly bounded. Then, the BV bound for $u \mapsto \mathcal{H}\left(x_{u}^{\varepsilon}(t, u)\right)$ leads to the BV bound for $u \mapsto x_{u}^{\varepsilon}(t, u)$.

Step 3. Furthermore, $x_{u}^{\varepsilon}$ is uniformly Lipschitz continuous from $[0, T] \mapsto$ $\mathbf{L}^{1} \overline{([a, b])}$ because the traveling speed for $x_{u}^{\varepsilon}$ is bounded. Indeed, on any interval $\left[u_{i}, u_{i+1}\right]$, we have

$$
\frac{d}{d t} x_{u}^{\varepsilon}=\frac{d}{d t}\left(\frac{1}{w_{i+\frac{1}{2}}(t)}\right)=-\frac{1}{w_{i+\frac{1}{2}}^{2}} \dot{w}_{i+\frac{1}{2}}(t)=-\frac{1}{w_{i+\frac{1}{2}}^{2}} f^{2}\left(w_{i+\frac{1}{2}}\right) F_{i+\frac{1}{2}}<\infty .
$$

Step 4. Combining step 2 and 3, by standard compactness argument we have $x_{u}^{\varepsilon} \rightarrow x_{u}$ in $\mathbf{L}_{l o c}^{1}([0, T] \times \mathbb{R})$. Furthermore, this imples $x^{\varepsilon} \rightarrow x$ for a.e. $t$ and all $u^{\varepsilon} \in \mathbb{R}$.

Step 5. Now, define the function

$$
\mathcal{G}(s) \doteq g\left(\frac{1}{s}\right)=s f\left(\frac{1}{s}\right) .
$$

Then, $\mathcal{G}(s)$ is Lipschitz for $s \in\left[0,1 / \kappa_{0}\right]$ because

$$
\mathcal{G}^{\prime}(s)=f\left(\frac{1}{s}\right)-\frac{1}{s} f^{\prime}\left(\frac{1}{s}\right)<\infty, \quad \frac{1}{s} \geq \kappa_{0} .
$$

Therefore, the convergence of $x_{u}^{\varepsilon}$ implies that $\mathcal{G}\left(x_{u}^{\varepsilon}(t, u)\right) \rightarrow g\left(x_{u}(t, u)\right)$ a.e., and also in $\mathbf{L}_{l o c}^{1}([0, T] \times \mathbb{R})$.

Step 6. Now, define the flux $F^{\varepsilon}(t, u)$ as

$$
F^{\varepsilon}(t, u) \doteq \exp \left\{\int_{u}^{\infty} \mathcal{G}\left(x_{u}^{\varepsilon}(t, s)\right) d s\right\}
$$

The map $u \mapsto F^{\varepsilon}(t, u)$ is Lipschitz because

$$
\left|\frac{d}{d u} F^{\varepsilon}(t, u)\right|=\left|\mathcal{G}\left(x_{u}^{\varepsilon}\right) F^{\varepsilon}\right|<\infty, \quad t \leq T .
$$

Therefore, we have that

$$
F^{\varepsilon}(t, u) \rightarrow F(t, u)=\exp \int_{u}^{\infty} \mathcal{G}\left(x_{u}(t, v)\right) d v, \quad \text { for a.e. } t \text { and all } u \in \mathbb{R} .
$$

Step 7. Returning back to the original variable $x$, for fixed $t \in[0, T]$, we know $u^{\varepsilon}(t, x) \rightarrow u(t, x)$ for a.e $x$ and in $\mathbf{L}_{l o c}^{1}$. Then, the map $x \rightarrow F^{\varepsilon}(t, x)$ is $\mathrm{BV}$, therefore $F^{\varepsilon}(t, x) \rightarrow F(t, x)$ for a.e. $x$, and $F^{\varepsilon}(t, x) \rightarrow F(t, u(t, x))$ 
at every point $x$ where $u$ is continuous, hence a.e. and in $\mathbf{L}_{l o c}^{1}$. Note the function $F(t, u(t, x))$ is defined as

$$
F(t, u(t, x))=\exp \int_{u}^{\infty} g\left(u_{x}(t, s)\right) d s=\exp \int_{x}^{\infty} f\left(u_{x}(t, y)\right) d y .
$$

Combined with $\mathbf{L}^{1}$ Lipschitz continuity in $t$, we conclude that $F^{\varepsilon}(t, x) \rightarrow$ $F(t, x)$ in $\mathbf{L}_{l o c}^{1}([0, T] \times \mathbb{R})$. This proves $(\mathrm{c} 4)$, therefore the existence of weak solutions.

\subsection{Discrete entropy inequality - Existence of entropy weak solutions with additional assumption (1.21).}

It remains to show that, as $\varepsilon \rightarrow 0$, all the singularities in the limit function are admissible. It suffices to show that the entropy inequality (3.34) holds in the limit. where

Define the discrete entropy and discrete entropy flux pair $\left(P^{\varepsilon}, Q^{\varepsilon} F^{\varepsilon}\right)$

$$
P^{\varepsilon}(t, x) \doteq P\left(u^{\varepsilon}(t, x), w^{\varepsilon}(t, x)\right), \quad Q^{\varepsilon}(t, x) \doteq Q\left(u^{\varepsilon}(t, x), w^{\varepsilon}(t, x)\right) .
$$

Recall that the functions $P$ and $Q$ are introduced in (3.19) and (3.21). The constants $(k, l) \in \Omega^{\varepsilon}$ are arbitrary, with

$$
\Omega^{\varepsilon} \doteq\left\{(k, l): u_{0}(0) \leq k \leq u_{N}(T), \zeta\left(\kappa_{0}\right) \leq l \leq \zeta(+\infty)\right\} .
$$

Discrete entropy inequality. Let $x_{i}$ be a nodal point, which is a singularity in the approximation. We use the same notations as in Lemma 6 , i.e., we use super-script - and + to denote the left and right state of the singulairty. If $x_{i}$ is a convex kink or the left of a shock, by Lemma 6 the entropy is dissipative, and (3.23) holds.

If $x_{i}$ is a concave kink with $w_{i+\frac{1}{2}}<w_{i-\frac{1}{2}}$, then it is small. When $k \leq u_{i}$ and $l>\zeta\left(w_{i+\frac{1}{2}}\right)$, the error introduced is bounded by a quadratic term of the wave strength. We have

$$
\begin{aligned}
& \left(P^{\varepsilon,+}-P^{\varepsilon,-}\right) \dot{x}_{i}-\left(Q^{\varepsilon,+}-Q^{\varepsilon,-}\right) F_{i} \\
\geq & -C\left(\zeta\left(w_{i-\frac{1}{2}}\right)-\zeta\left(w_{i+\frac{1}{2}}\right)\right)^{2} \geq-C\left(\zeta\left(w_{i-\frac{1}{2}}\right)-\zeta\left(w_{i+\frac{1}{2}}\right)\right) \varepsilon .
\end{aligned}
$$

Here in the last inequality we used the estimate (5.29).

If $x_{i}$ is at the right side of a shock, then, the Lax condition is only approximated. For $u_{i-1}<k<u_{i}$ and $l>\zeta\left(w_{i+\frac{1}{2}}\right)$, we have

$$
\begin{aligned}
& \left(P^{\varepsilon,+}-P^{\varepsilon,-}\right) \dot{x}_{i}-\left(Q^{\varepsilon,+} F_{i}-Q^{\varepsilon,-} F_{i-1}\right) \\
\geq & -C\left(f^{\prime}\left(w_{i+\frac{1}{2}}\right) F_{i}-\dot{x}_{i}\right)^{2} \geq-C\left(f^{\prime}\left(w_{i+\frac{1}{2}}\right) F_{i}-\dot{x}_{i}\right) \varepsilon \\
\geq & -C F_{i}\left(f^{\prime}\left(w_{i+\frac{1}{2}}\right)-\eta_{0}\right) \varepsilon \geq-C\left(\zeta(+\infty)-\zeta\left(w_{i+\frac{1}{2}}\right)\right) \varepsilon .
\end{aligned}
$$


Here we used the condition (4.18), the property (3.6) and the uniform estimate on $F$.

For any approximate solution $u^{\varepsilon}, w^{\varepsilon}$, we have the following discrete entropy inequality: for any smooth test function $\varphi \geq 0$, and any constants $(k, l) \in \Omega^{\varepsilon}$, it holds

$$
\begin{aligned}
& \int_{0}^{T} \int_{\mathbb{R}} P^{\varepsilon} \varphi_{t}+Q^{\varepsilon} F^{\varepsilon} \varphi_{x} d x d t+\int_{0}^{T} \int_{\mathbb{R}} \mathcal{S}\left(u^{\varepsilon}, w^{\varepsilon}\right) F^{\varepsilon} \varphi d x d t \\
& -\int_{\mathbb{R}}\left(P^{\varepsilon}(T, x) \varphi(T, x)-P^{\varepsilon}(0, x) \varphi(0, x)\right) d x \geq-\left(e_{1}+e_{2}\right) .
\end{aligned}
$$

We recall that $\mathcal{S}$ is the source term defined in (3.32). Here $e_{1}$ and $e_{2}$ are error terms. The term $e_{1}$ includes all errors caused by small concave kinks and the approximated Lax condition on the right of a shock. Thanks to (6.7) and (6.8), we have

$$
e_{1} \leq C T \varepsilon \cdot \operatorname{TV}\left\{\zeta^{\varepsilon}\right\} \leq C \varepsilon .
$$

because $\zeta^{\varepsilon}$ is $\mathrm{BV}$, see (5.22). Then, $e_{1}$ vanishes as $\varepsilon \rightarrow 0$.

The term $e_{2}$ contains error caused by the approximation of $F^{\varepsilon}$ by the linear interpolation $\widehat{F}^{\varepsilon}$. We have

$$
e_{2}=\int_{0}^{T} \int_{\mathbb{R}}\left|F^{\varepsilon}-\widehat{F}^{\varepsilon}\right| \mathcal{S}\left(u^{\varepsilon}, w^{\varepsilon}\right) \varphi d x d t .
$$

Convergence. Thanks to the a priori bounds on $u^{\varepsilon}, w^{\varepsilon}$, both the discrete entropy $Q^{\varepsilon}$ and the discrete entropy flux $Q^{\varepsilon}$ are uniformly bounded and BV functions on any bounded set. Therefore, as $\varepsilon \rightarrow 0$, we have

$$
P^{\varepsilon} \rightarrow P(u, w), \quad Q^{\varepsilon} \rightarrow Q(u, w), \quad \text { in } \mathbf{L}_{l o c}^{1} .
$$

This implies the convergence of the first and the third terms on the left hand side of (6.9).

The convergence of the second term follows from the fact that the map $w \rightarrow \mathcal{S}$ is uniformly bounded for $w \geq \kappa_{0}$. Consequently, the error term $e_{2}$ vanishes as $\varepsilon \rightarrow 0$.

In conclusion, as $\varepsilon \rightarrow 0$, the limit solution satisfies the entropy inequality (3.34), which is equivalent to the Lax entropy condition. This proves Theorem 1 with the additional assumption (1.21).

\subsection{Existence of entropy weak solutions; Proof of the main Theorem.}

We now show that the additional assumption (1.21) can be dropped.

Let $f$ be a function that satisfies the assumptions $(\mathbf{A})$. We construct a sequence of approximate function $f_{n}$, such that $f_{n}$ satisfies the assumptions (A) and the additional assumption (1.21). This could be achieved by taking (for $n>3$ )

$$
f_{n}(w)= \begin{cases}f(n)+\eta_{0}(w-n), & w \geq n \\ f(w), & w \leq n-1\end{cases}
$$


and connecting the two parts smoothly through the interval $w \in(n-1, n)$ such that $f_{n}$ is $\mathcal{C}^{2}$ and convex.

Clearly, as $n \rightarrow+\infty$ we have

$$
f_{n} \rightarrow f, \quad f_{n}^{\prime} \rightarrow f^{\prime}, \quad \text { uniformly on }\left[\kappa_{0},+\infty\right) .
$$

Introducing the function $g_{n}$

$$
g_{n}(w) \doteq \frac{1}{w} f_{n}(w),
$$

then, $g_{n}$ is uniformly bounded on $\left[\kappa_{0}, \infty\right)$, and

$$
g_{n} \rightarrow g, \quad \text { uniformly on }\left[\kappa_{0},+\infty\right) .
$$

Let $u_{n}$ be an entropy weak solution of (1.1) with the erosion function $f_{n}$, constructed as the limit of the piecewise affine approximations. The goal now is to show that, in the limit as $n \rightarrow+\infty, u_{n}$ converges to an entropy weak solution for (1.1) with the erosion function $f$.

The proof can be carried out in a similar way as for the convergence of the approximate solution $u^{\varepsilon}$, since the sequence $\left\{u_{n}\right\}$ shares the same a priori bounds as those for $\left\{u^{\varepsilon}\right\}$. We follow the same steps as in Section 6.1. The properties (c1) and (c2) follow, and here we have no error term so (c3) also holds. limit,

The key step is (c4), i.e, the global flux should converges to the correct

$$
\int_{x} f_{n}\left(\left(u_{n}\right)_{x}\right) d y \rightarrow \int_{x} f\left((u)_{x}\right) d y .
$$

Introducing the function $\mathcal{G}_{n}$, similar to (6.5)

$$
\mathcal{G}_{n}(s) \doteq g_{n}\left(\frac{1}{s}\right) \doteq s f_{n}\left(\frac{1}{s}\right) .
$$

The convergence of $g_{n}$ in (6.14) now implies the convergence for $\mathcal{G}_{n}$,

$$
\mathcal{G}_{n} \rightarrow \mathcal{G} \quad \text { uniformly on }\left(0,1 / \kappa_{0}\right] .
$$

Therefore,

$$
\mathcal{G}_{n}\left(\frac{1}{\left(u_{n}\right)_{x}}(t, u)\right) \rightarrow \mathcal{G}\left(\frac{1}{u_{x}}(t, u)\right) \quad \text { a.e. . }
$$

The result follows in the same way as in the rest of Section 6.1, leading to existence of weak solutions.

Writing $\left(P_{n}, Q_{n} F_{n}\right)$ as the entropy pair, they satisfy the inequality (6.9) with $e_{1}=e_{2}=0$. The convergence of this inequality follows in a same way as that of $\left(P^{\varepsilon}, Q^{\varepsilon} F^{\varepsilon}\right)$. This completes the proof of Theorem 1 . 


\section{Concluding Remarks}

In this paper we establish the global existence of BV solutions for a non-local model describing slow erosion, also in cases where the slope of the mountain profile blows up. However, the uniqueness and continuous dependence of solutions remains an open problem. The main difficulty lies in the fact that, when $u$ is discontinuous, $u_{x}$ is a distribution containing point masses. Hence one cannot apply standard techniques to the conservation law (2.2) describing the evolution of $u_{x}$. Well posedness of the equation (1.1) will be a matter for future investigation.

Acknowledgements. The work of the first author is partially supported by an NSF grant no DMS-0908047.

\section{References}

1. Amadori, D. And Shen, W.: Global Existence of large BV solutions in a Model of Granular Flow. Comm. PDE. 34, 1003-1040 (2009).

2. Amadori, D. And Shen, W.: The Slow Erosion Limit in a Model of Granular Flow, Arch. Ration. Mech. Anal. 199, 1-31 (2011).

3. Amadori, D. And Shen, W.: An Integro-Differential Conservation Law arising in a Model of Granular Flow. To appear in Journal of Hyperbolic Differential Equations.

4. Amadori, D. And Shen, W.: Front Tracing Approximations for Slow Erosion. To appear in Discrete and Continuous Dynamical System -A.

5. Boutreux, T. And Gennes, P.-G.: Surface flows of granular mixtures, I. General principles and minimal model. J. Phys. I France 6, 1295-1304 (1996).

6. Bressan, A.: Hyperbolic Systems of Conservation Laws. The One Dimensional Cauchy Problem. Oxford University Press, 2000.

7. Bressan, A. And Constantin, A.: Global dissipative solutions of the Camassa-Holm equation. Anal. Appl. (Singap.) 5, 1-27 (2007).

8. Bressan, A. And Constantin, A.: Global conservative solutions of the Camassa-Holm equation. Arch. Ration. Mech. Anal. 183, 215-239 (2007).

9. Bressan, A. And Zhang, P. And Zheng, Y.: Asymptotic variational wave equations. Arch. Ration. Mech. Anal. 183, 163-185 (2007).

10. Camassa, R. AND Holm, D.: An integrable shallow water equation with peaked solitons. Phys. Rev. Lett. 71, 1661-1664 (1993).

11. Cannarsa, P. and Cardaliaguet, P.: Representation of equilibrium solutions to the table problem for growing sandpiles. J. Eur. Math. Soc. (JEMS) 6, 435-464 (2004).

12. Cannarsa, P. and Cardaliaguet, P. and Crasta, G. and Giorgieri, E.: A boundary value problem for a PDE model in mass transfer theory: representation of solutions and applications. Calc. Var. Partial Differential Equations 24, 431-457 (2005).

13. Colombo, R. and Guerra, G. and Monti, F.: Modeling the Dynamics of Granular Matter. To appear in IMA J. Appl. Math.

14. Colombo, R.M., Mercier, M. and Rosini M.: Stability and total variation estimates on general scalar balance laws. Commun. Math. Sci. 7, 37-65 (2009).

15. Dafermos, C.M.: Hyperbolic Conservation Laws in Continuum Physics. (3rd Ed.) Springer, Heidelberg, 2010.

16. Duran, J.: Sands, Powders, and Grains: An Introduction to the Physics of Granular Materials, Springer-Verlag, 2000. 
17. Falcone, M. And Finzi Vita, S.: A finite-difference approximation of a two-layer system for growing sandpiles. SIAM J. Sci. Comput. 28, 1120-1132 (2006).

18. Hadeler, K.P. And Kuttler, C.: Dynamical models for granular matter. Granular Matter 2, 9-18 (1999).

19. Hunter, J.K. And Zheng, Y.: On a nonlinear hyperbolic variational equation I and II. Arch. Ration. Mech. Anal. 129, 305-383 (1995).

20. LAx, P.: Hyperbolic systems of conservation laws II. Commun. Pure Appl. Math. 10, 537-566 (1957).

21. LiU, T.P.: Admissible solutions of hyperbolic conservation laws. Mem. Amer. Math. Soc. 30 no. 240 (1981).

22. Oleinik, O.A.: Discontinuous Solutions of non-linear differential equations. Usp. Mat. Nauk. 12, 3-73 (1957). English translation: AMS Translations, Ser. II, 26, 95-172.

23. Savage, S.B. And Hutter, K.: The dynamics of avalanches of granular materials from initiation to runout. I. Analysis. Acta Mech. 86, 201-223 (1991).

24. Shen, W.: On the Shape of Avalanches. J. Math. Anal. Appl. 339, 828-838 (2008).

Wen Shen

Department of Mathematics, Penn State University, U.S.A. email: shen_w@math.psu.edu

and

Tianyou Zhang

Department of Mathematics, Penn State University, U.S.A.

email: zhang_t@math.psu.edu 